UNIVERSIDADE DE SÃO PAULO

FACULDADE DE ODONTOLOGIA DE RIBEIRÃO PRETO DEPARTAMENTO DE ODONTOLOGIA RESTAURADORA

JOSÉ MENDES DA SILVA

\title{
INFLUÊNCIA DO TORO MANDIBULAR NA ATIVIDADE ELETROMIOGRÁFICA DOS MÚSCULOS DA MASTIGAÇÃO
}




Músculos da Mastigação

Tese apresentada à Faculdade de Odontologia de Ribeirão Preto da Universidade de São Paulo para obtenção do grau de Doutor em Odontologia Restauradora

Área de Concentração: Odontologia Restauradoraopção: Dentística

Orientadora: Profa. Dra. Simone Cecilio Hallak Regalo 
AUTORIZO A REPRODUÇÃO E/OU DIVULGAÇÃO TOTAL OU PARCIAL DA PRESENTE OBRA POR QUALQUER MEIO CONVENCIONAL OU ELETRÔNICO, PARA FINS DE ESTUDO E PESQUISA, DESDE QUE CITADA A FONTE.

FICHA CATALOGRÁFICA

Da Silva, José Mendes.

Influência do toro mandibular na atividade eletromiográfica dos músculos da mastigação. Ribeirão Preto, 2014.

86 p.; $30 \mathrm{~cm}$

Tese de Doutorado apresentada a Faculdade de Odontologia de Ribeirão Preto da Universidade de São Paulo

Orientadora: Regalo, Simone Cecilio Hallak.

1. Músculos mastigatórios 2. Eletromiografia

3. Toro Mandibular 


\section{FOLHA DE APROVAÇÃO}

Candidato: José Mendes da Silva

Título da Tese: Influência do Toro Mandibular na Atividade Eletromiográfica dos Músculos da Mastigação

A comissão julgadora dos trabalhos de defesa da tese de Doutorado, Faculdade de Odontologia de Ribeirão Preto da Universidade de São Paulo, em sessão pública realizada a................/2014, considerou o candidato...................................

\section{BANCA EXAMINADORA}

1. Prof. Dr. Instituição:

Julgamento: Assinatura:

2. Prof. Dr. Instituição:

Julgamento: Assinatura:

3. Prof. Dr. Instituição:

Julgamento: Assinatura:

4. Prof. Dr. Instituição:

Julgamento: Assinatura:

5. Prof. Dr. Instituição: Assinatura: 



\section{DEDICATÓRIA}

\section{Aos Meus Pais Dinoral Mendes da Silva (In Memória) e Clotildes Umbelina Mendes}

Agradeço pela minha formação, pelos valores éticos e morais que me transmitiram, pelo que me propiciaram, educação, bem estar e condições de realizar meu sonho que foi a graduação em Odontologia.

A vocês, minha eterna gratidão.

\section{À Minha esposa Maria Gislene Angélica de Brito e Mendes}

Obrigado pelo apoio incondicional, pelo carinho, dedicação, sempre ao meu lado. Tivemos momentos maravilhosos e momentos muito difíceis, mas juntos superamos os obstáculos que apareceram no nosso caminho.

Agradeço a Deus por ter feito com que nossos caminhos se cruzassem e unisse nossas vidas para sempre.

\section{Aos meus filhos Laíse, Hugo e Fernanda}

Meus filhos quando chegaram floriram nosso lar, os ensinamentos dirigidos a eles retornam diariamente em aprendizado e em incentivo para a minha constante busca do conhecimento. 



\section{AGRADECTMENTOS ESPECAAIS}

\section{A DEUS}

O Grande Arquiteto do Universo, que criou todas as coisas, que a tudo vê e nos conduz para o caminho do bem. É o Responsável pela existência de um mundo harmonioso, repleto de maravilhas e oportunidades a nossa disposição.

Agradeço ao dom da vida, às oportunidades que me foram dadas e por me permitir alcançar tantas conquistas durante minha caminhada.

\section{À minha Orientadora Profa. Dra. Simone Cecilio Hallak Regalo}

Educadora por excelência, com a sua sensibilidade orienta os alunos respeitando a individualidade de cada um e explorando suas potencialidades. Professora dedicada, com um jeito suave e firme transmite confiança, norteando os passos de seus orientandos na pesquisa, de forma a ensiná-los a fazer ciência e conduzindo-os na construção do conhecimento.

Profa. Simone, sua orientação foi fundamental para a conquista deste tão almejado título. Você contribuiu de forma efetiva na minha qualificação profissional.

\section{Aos Professores da USP Ribeirão Preto}

Agradeço a todos os professores do Programa por acreditarem e tornarem realidade este Doutorado Interinstitucional, deslocando-se quilômetros de distância, para transmitir conhecimento e contribuir com a qualificação do corpo docente da Universidade Estadual de Montes Claros.

Minha gratidão a todos vocês.

\section{À Laíse Angélica Mendes Rodrigues}

Minha filha, MS em Odontologia, que me ajudou na elaboração dessa Tese. 



\section{AGRADECTMENTOS}

À Faculdade de Odontologia de Ribeirão Preto da Universidade de São Paulo e à Universidade Estadual de Montes Claros agradeço o empenho que tiveram para estabelecer a parceria que viabilizou o DINTER em Odontologia Restauradora. Agradecimento especial ao Prof. Dr. Manoel Damião Souza Neto e Prof. Dr. Marcelo Oliveira Mazzetto, coordenador e vice coordenador do programa Dinter.

Ao Centro de Educação Profissional e Tecnológica da UNIMONTES agradeço por ceder espaço para construção e instalação do Laboratório de Pesquisa em Odontologia, que foi essencial para nossa qualificação.

Aos colegas do Doutorado, Manoel Brito, Carla, Cássia Pérola, Deícola, Agnaldo, Tânia, Soraya, Adriane e Altair pelo companheirismo e solidariedade nos momentos difíceis, dividindo angústias e vislumbrando caminhos.

Ao Paulo Batista de Vasconcelos MS da USP/Ribeirão Preto pela sua competência e desprendimento na realização dos exames para coleta dos dados para este estudo. 

“Ninguém caminha sem aprender a caminhar, sem aprender a fazer o caminho caminhando, refazendo e retocando o sonho pelo qual se pôs a caminhar."

\section{Paulo Freire}

"O que vale na vida não é o ponto de partida e sim a caminhada. Caminhando e semeando, no fim terás o que colher." 



\section{RESUMO}

Da Silva, JM. Influência do toro mandibular na atividade eletromiográfica dos músculos da mastigação (tese). Ribeirão Preto: Faculdade de Odontologia de Ribeirão Preto da Universidade de São Paulo; 2014.

O toro é uma excrescência óssea convexa, com crescimento lento, considerado de origem congênita, benigno, também denominado de exostose, com pouca significância clínica, sendo necessária sua remoção quando dificulta a confecção de prótese totais, parciais ou removíveis e a posição da língua, interferindo na fala ou ainda, dificultam a intubação cirúrgica. O objetivo deste trabalho foi analisar, por meio da eletromiografia, o padrão de comportamento (morfológico e funcional) da atividade dos músculos masseter e temporal nas condições posturais da mandíbula como o Repouso, Protrusão, Máxima Interscupidação Habitual (MIH), Apertamento Dental com Parafilme $\mathrm{M}^{\circledR}$, Lateralidades Direita e Esquerda. O presente estudo também propôs analisar a eficiência dos ciclos mastigatórios e para tanto, foi utilizado a avaliação da mastigação habitual e não habitual por meio da integral da envoltória do sinal eletromiográfico obtido dos músculos masseter e temporal durante a mastigação habitual de uvas passas e amendoim e da mastigação não-habitual de Parafilme $\mathbf{M}^{\circledR}$, considerando a presença ou não do toro mandibular. Diante dos resultados obtidos no presente trabalho foi possível concluir que, de um modo geral, houve aumento na atividade eletromiográfica nos músculos masseteres de indivíduos portadores de toro mandibular (Grupo Casos) quando comparado aos indivíduos do Grupo Controles nas condições clínicas de protrusão, lateralidades direita e esquerda, máxima intercuspidação habitual (MIH), apertamento dental com parafilme $M^{\circledR}$. Na mastigação com parafilme $M^{\circledR}$, mastigação com amendoins e mastigação com uvas passas verificou-se aumento da atividade para os músculos temporais e masseteres, evidenciando que a presença de toro mandibular está associada a alterações no padrão de ativação da musculatura mastigatória nas condições posturais da mandíbula e na mastigação.

Palavras-Chave: Toro mandibular, Eletromiografia, Sistema estomatognático. 



\begin{abstract}
The torus is a convex bony outgrowth, considered congenital, benign, also called exostosis, with little clinical significance, necessitating its removal difficult when the making of complete dentures, removable partial or tongue position and interfering speech or hinder surgical intubation. The aim of this study was to analyze, using electromyography, the default behavior (morphological and functional) of activity of the masseter and temporal muscles in conditions mandible posture as Home, protrusion, Maximum Interscupidação Habitual (MIH), Dental Clenching with Parafilm $\mathbf{M}^{\circledR}$, lateral excursion (right and left). This study also proposed to analyze the efficiency of the mastigatory cycles. Therefore, we used the evaluation of usual mastication and unusual mastication through the envoltory integral of the EMG signal obtained from the masseter and temporalis muscles during mastication of raisins and peanuts and unusual chewing Parafilm $\mathrm{M}^{\circledR}$, considering the presence or no in mandibular torus. Given the results obtained in this study it was concluded that, in general, increased the EMG activity in the masseter muscles of patients with mandibular torus (Group Case) when compared to individuals of the Group Controls in the mandible clinical conditions as protrusion, lateral excursion (right and left), maximum intercuspation (MIH), clenching with parafilm $\mathrm{M}^{\circledR}$. There was an increase in EMG activity of the temporal and masseter muscles during mastication with parafilm $\mathrm{M}^{\circledR}$, mastication with peanuts and mastication with raisins, showing that the presence of mandibular torus is associated with changes in the default activation of masticatory muscle in conditions mandible posture and chewing.
\end{abstract}

Keyword: Toro mandibular Electromyography, stomatognathic system. 



\section{SUMÁRIO}

INTRODUÇÃO .................................................................................................................22

PROPOSIÇÃO ...................................................................................................................................

MATERIAL E MÉTODOS ..............................................................................................35

RESULTADOS .............................................................................................................................4

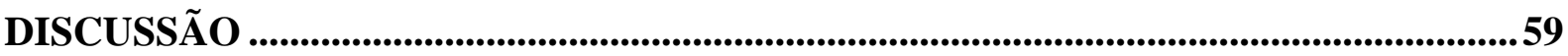

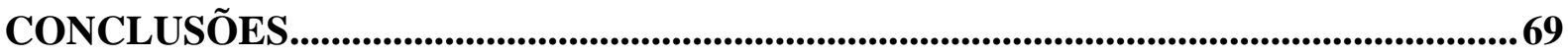

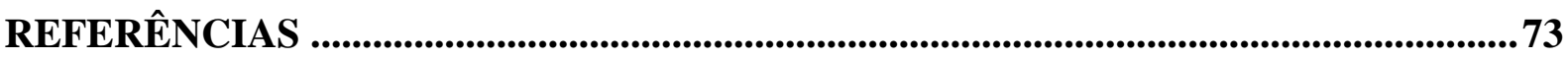

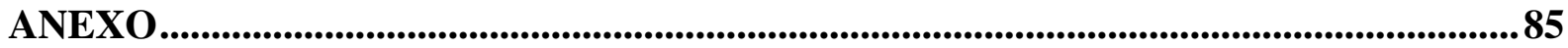





\section{INTRODUÇÃO}





\section{INTRODUÇÃO}

Exostoses são protuberâncias ósseas localizadas, que apresentam tamanho variável, se originam da cortical óssea, se manifestam em diversas regiões do corpo e são consideradas variações anatômicas da normalidade. Podem ocorrer durante o período embrionário, mas normalmente, ficam aparentes na segunda ou terceira década de vida. Na cavidade bucal podem aparecer diversos tipos de exostoses, entre as quais, as mais comuns são o toro palatino (TP) e o toro mandibular (TM), localizados na linha média do palato duro e ao longo da superfície lingual da mandíbula, respectivamente (FURTADO et al., 2008).

O termo toro designa excrescência óssea convexa bem definida de crescimento lento, composto por uma densa cortical, escassa em osso esponjoso, coberta por uma capa de mucosa delgada e pobremente irrigada (PINZÓN et al., 2007; MORRISON; TAMIMI, 2012). É considerado congênito, benigno sendo denominado também de exostose, do grego exo=fora e osteo=osso, apresentando pequena significância clínica, mas em alguns casos com necessidade de remoção por dificultar a confecção de prótese totais, parciais ou removíveis (PONZONI et al., 2008) e a posição da língua, interferindo na fala (SPRINGER, 1954; SHIMAHARA et al., 2007; MARTINS et al., 2007) ou ainda dificultam a intubação cirúrgica (ANTONIADES et al., 1998; TAKASUGI et al., 2009; HASSAN et al., 2012).

Segundo Kolas et al. (1953) o toro mandibular pode ser classificado como unilateral único, unilateral múltiplo, bilateral único e múltiplo e apresenta normalmente forma arredondada, superfície lisa, projeção de osso duro e coberto por mucosa normal (REGEZI, SCIUBBA; 2000).

O toro mandibular é bilateral em 90\% dos casos, da maioria das vezes a lesão é assintomática sendo percebida apenas quando algum trauma lesiona a mucosa e causa desconforto, quando da necessidade de reabilitação oral ou em caso de cirurgia (NEVILLE et al, 2004). 
Quando radiografado, o toro mandibular apresenta imagem circunscrita, radiodensa e de alta sobreposição de radiopacidade na raiz dos dentes inferiores, geralmente os pré-molares (RENON et al.,1994; REGEZI, SCIUBBA; 2000; NEVILLE et al., 2004).

Histologicamente o toro mandibular se assemelha ao osso normal, composto de osso hiperplásico, estrutura compacta e de uma estrutura central esponjosa com espaços medulares bem definidos (NEVILLE et al, 2004).

O toro pode localizar-se na maxila na linha mediana, quando presente na mandíbula, surge entre suas tábuas internas, assim como pode surgir em qualquer outra parte do esqueleto, sendo mais frequente nas diáfises dos ossos longos e em áreas de reparação de fraturas (PINZÓN et al., 2007). O toro é considerado anomalia de desenvolvimento rara em crianças menores de 10 anos (BERNABA, 1977), mas frequentemente observado em adultos jovens e indivíduos de meia idade (SIRIRUNGROJYING, KERDPON, 1999).

A ocorrência e a coexistência de exostoses e toro têm sido investigadas por vários autores há muito tempo (SIRIRUNGROJYING, KERDPON, 1999; KERDPON, SIRIRUNGROJYING, 1999; NASCIMENTO FILHO et al.,2004; BUKHARI et al., 2007). A ocorrência do toro em vários grupos étnicos pode variar de 0,5\% em índios brasileiros (BERNABA, 1977), 3,2 \% em nigerianos (DOSUMU et al., 1998), 20\% em brasileiros (FURTADO et al., 2008), 31,9\% em tailandeses (APINHASMIT et al., 2002) até 74\% em japoneses (IGARASHI et al., 2008). Alguns autores acreditam que o toro palatino é mais frequente em mulheres (BUKHARI et al., 2007; GARCÍA-GARCÍA et al., 2010) e o toro mandibular é mais frequente em homens (EGGEN,1989).

Um estudo epidemiológico de lesões diagnosticadas durante vinte e nove anos verificou que a idade média de indivíduos com toro é 43,66 anos, 60\% aparece no gênero feminino e a localização mandibular é preferencial (GRANDI et al.,2005). 
A etiologia do toro ainda permanece obscura, embora tenham sido apresentadas evidências que sugerem que o toro pode ser uma condição hereditária (REGEZI, SCIUBBA, 2000; AL-BAYATY et al.,2001). Tem sido considerada multifatorial e é atribuída a fatores genéticos, ambientais, hiperfunção mastigatória e crescimento continuado (SIRIRUNGROJYING, KERDPON, 1999; BUKHARI et al., 2007).

Recentemente, alguns trabalhos sugeriram uma relação com disfunção temporomandibular (SIRIRUNGROJYING, KERDPON, 1999; FURTADO et al., 2008; SHANKLAND, 2010) e o bruxismo (SIRIRUNGROJYING, 1999; SERRA, CAMPOS, 2005; SERRA et al., 2005; IGARASHI et al., 2008) sem comprovarem efetivamente essa relação, mas Neville, et al (2004) realizaram uma revisão de literatura, mostrando que parece haver relação dos hábitos parafuncionais na etiologia do toro mandibular.

O estudo de Eggen e Natvig (1986) com 2000 indivíduos demonstraram uma relação entre a frequência de toro mandibular e as forças funcionais, bem como o número de dentes presentes. Furtado et al. (2008) relataram que o predomínio de toro mandibular está associado diretamente à presença dos dentes. Segundo os autores, o toro mandibular pode ser formado pelo choque das forças sobre as cúspides e a transmissão de força da face palatina do dente superior para a face lingual do processo alveolar. Esta afirmação vai ao encontro das pesquisas de outros autores (KERDPON, SIRIRUNGROJYING, 1999; ÇAGIRANKAYA et al., 2005; NAKAMURA et al.,2007) que sugeriram que o estresse mastigatório pode ser a maior causa do desenvolvimento do toro e este por sua vez pode ser um indicador de atividade parafuncional.

Clifford e Fartash (1996) encontraram uma prevalência mais alta de toro mandibular em indivíduos portadores de disfunção temporomandibular, semelhante às afirmações de Pechenkina e Benfer (2002) de que existe uma correlação significante entre toro mandibular, exostoses e patologias da articulação temporomandibular. 
Pode existir uma associação entre toro mandibular e a presença de facetas de desgaste, portanto o grau de atrição dental é um dos indicadores que os dentes e a mandíbula sofrem estresse oclusal, mostrando que o estresse mastigatório é responsável pelo desenvolvimento do toro (IGARASHI et al.,2008).

Furtado et al. (2008) verificaram a associação de exostoses e/ou toro palatino e/ou mandibular com as variantes gênero, idade, raça, localização e presença de disfunção temporomandibular. Dos 75 indivíduos examinados, 82,4\% (n=61) eram portadores de mialgia e 17,6\% (n=13) apresentaram disfunções intracapsulares. Dos indivíduos portadores de disfunção intracapsulares, 76,9\% $(n=10)$ apresentaram deslocamento do disco com redução; 15,4\%(n=2) apresentaram subluxação; 7,7\% $(n=1)$ eram portadores de adesão de superfície e não observou-se nenhum caso com deslocamento do disco sem redução e desvio de forma.

Atividades de parafunções podem ser só coincidentes na etiologia de disfunção temporomandibular (DTM) e toro mandibular (TM). Porém, deveriam se realizar estudos longitudinais e uma amostra maior deveria ser considerada para investigar o mecanismo de desenvolvimento de toro mandibular como um indicador de risco aumentado para os sinais de DTM em alguns indivíduos, assim como análises da atividade dos músculos envolvidos na mastigação por meio da eletromiografia.

A Odontologia tem cada vez mais utilizado uma associação de métodos como a ultrassonografia e a eletromiografia para diagnóstico e avaliação de tratamentos das disfunções crânio mandibulares, principalmente com relação ao sistema estomatognático, frente à crescente valorização do aspecto funcional deste sistema. Um tratamento só é considerado integral se as relações forem estáveis e se houver harmonia entre forma e função (REGALO et al., 2008, De ROSSI et al., 2013). A eletromiografia possibilita a consolidação do conhecimento da fisiologia como uma parte integrante dos conceitos de pesquisa na área 
da Odontologia. O desenvolvimento harmônico muscular é fator importante na função mastigatória, anatômica e funcional do sistema estomatognático (SIÉSSERE et al., 2009; CECILIO et al., 2010; BERSANI et al., 2011). O estudo eletromiográfico dos músculos da mastigação teve início com Moyers (1949) que relatou em seu trabalho que o exame eletromiográfico é um método de auxílio para obtenção de informações mais corretas ou precisas, no que se refere à atividade muscular. A partir daí, inúmeras pesquisas utilizam essa técnica para avaliar a função dos músculos mastigatórios em diferentes condições clínicas posturais da mandíbula (KÖNIG JR.,VITTI, 1974; VITTI, BASMAJIAN, 1977; PANCHERZ, 1980; GIBBS ET AL., 1984; JANKELSON, 1990).

Hermens et al. (1999) publicaram os resultados do projeto SENIAM (European Recommendations for Surface Electromyography), que padronizou o tipo de eletrodo, seu tamanho e material, as configurações, os locais de captação do sinal, as formas de processamento e o tipo de filtro que deveria ser utilizado na coleta dos sinais de eletromiografia de superfície. Okeson (2000) relatou que pequenas variações na colocação de eletrodos nos indivíduos mudam significantemente o sinal coletado e que cuidados devem ser tomados durante a colocação dos mesmos entre múltiplas visitas de coletas.

Segundo Regalo et al. (2008) e Cecílio et al., 2010, o exame eletromiográfico vem sendo utilizado como ferramenta auxiliar para facilitar diagnósticos e estabelecer prognósticos mais cuidadosos, mostrando ser mais que um elemento complementar para estudo anatômico, fisiológico e neurofisiológico do sistema músculo esquelético, além da fácil aplicação, não provocando desconforto ao indivíduo nem interferindo na fisiologia muscular local. 


\section{PROPOSIÇÃO}





\section{PROPOSIÇÃO}

O objetivo deste trabalho foi analisar, por meio da eletromiografia, o padrão de comportamento (morfológico e funcional) da atividade dos músculos masseter e temporal nas condições posturais da mandíbula como o Repouso, Protrusão, Máxima Interscupidação Habitual (MIH), Apertamento Dental com Parafilme $\mathbf{M}^{\circledR}$ e Lateralidades Direita e Esquerda. O presente estudo também propôs analisar a eficiência dos ciclos mastigatórios, utilizando a avaliação da mastigação habitual e não habitual por meio da integral da envoltória do sinal eletromiográfico obtido dos músculos masseter e temporal durante a mastigação habitual de uvas passas e amendoim e da mastigação não-habitual de Parafilme $\mathbf{M}^{\circledR}$, considerando a presença ou não do toro mandibular. 
MATERIAL E MÉTODOS 



\section{MATERIAL E MÉTODOS}

\section{Amostra}

De uma amostra inicial de 120 indivíduos, foram selecionados para participar desta pesquisa 40 indivíduos adultos com idade mínima de 20 anos, que foram divididos em dois grupos:

$\checkmark$ Grupo Casos: constituído por 20 indivíduos portadores de toro mandibular bilateral. $\checkmark$ Grupo Controles: constituído por 20 indivíduos sem toro mandibular.

Trata-se de uma amostra de conveniência, selecionada entre os estudantes, docentes e funcionários da Universidade de Montes Claros durante o primeiro semestre de 2013. Para selecionar os indivíduos do Grupo Casos, portadores de toro mandibular bilateral, a amostra foi avaliada por meio de anamnese e exame clínico inicial. Quando diagnosticada a presença do toro, executava-se uma radiografia oclusal para descartar outras lesões possíveis. Posteriormente o Grupo Casos foi pareado sujeito a sujeito, com base no gênero, peso e idade para constituir o Grupo controles.

\section{Anamnese}

A anamnese serviu para levantar os dados pessoais, o histórico médico, uso de medicamentos, tratamentos que estavam sendo realizados, histórico dental, presença de hábitos parafuncionais e possíveis sintomas clínicos de disfunção temporomandibular (Índice Clínico de Fonseca e o RDC/TMD). Não houve distinção de gênero ou classe social.

\section{Exame Clínico}

Todos os indivíduos da amostra foram examinados, identificando a presença ou não de toro mandibular bilateral, identificando se todos eram dentados completos ou reabilitados e se tinham ou não outras lesões e/ou tumores. 


\section{Exame Radiográfico}

Os indivíduos portadores de toros mandibular bilateral selecionados foram submetidos a exame radiográfico oclusal mandibular, para confirmação do diagnóstico de toro e exclusão de possíveis lesões e ou tumores, com aspecto clínico semelhante (Figura 1).

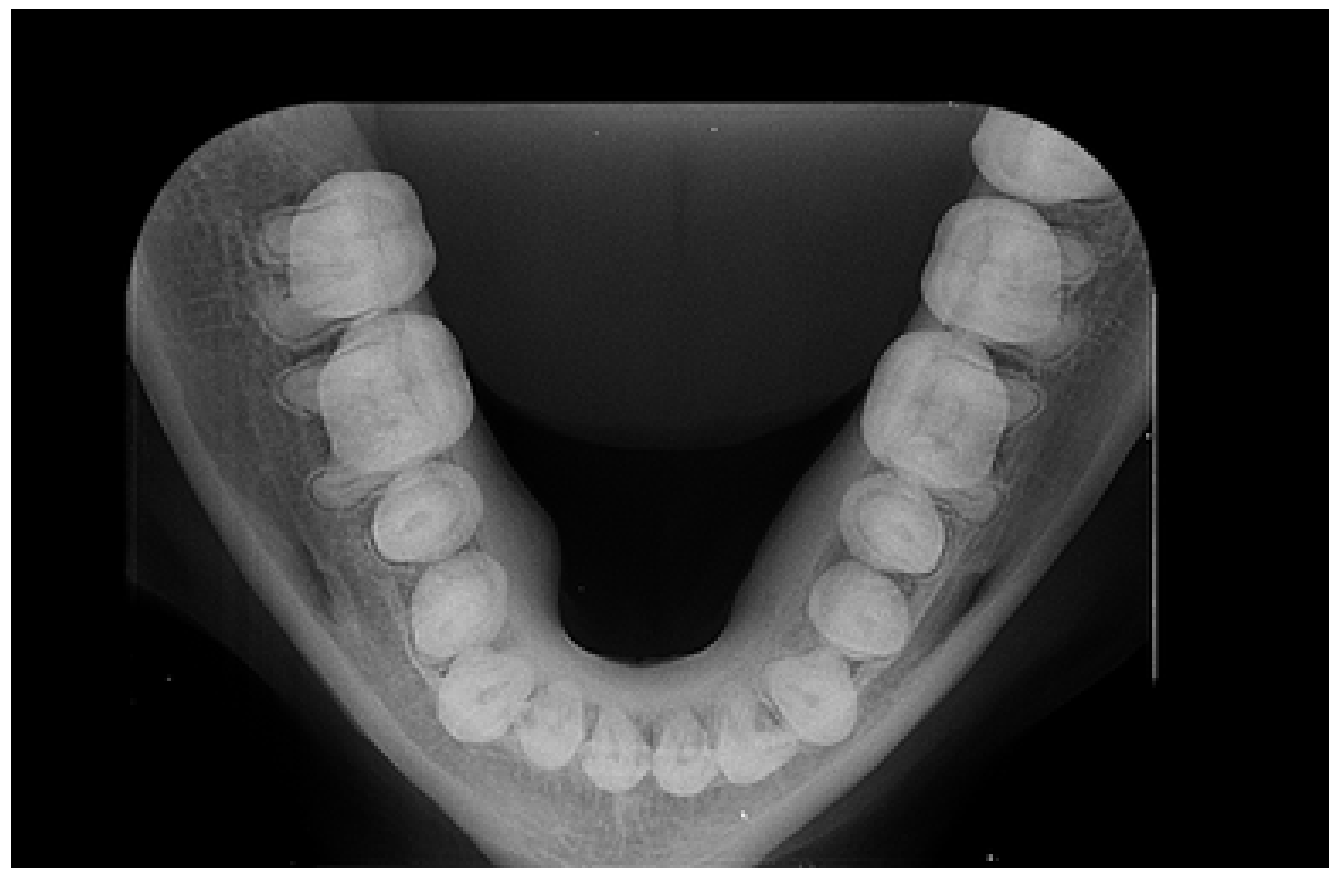

Figura 1. Radiografia oclusal de indivíduo portador de toro mandibular bilateral.

\section{Critérios de inclusão}

Foram incluídos na pesquisa indivíduos dentados completos ou reabilitados por meio de prótese fixa de no máximo um elemento, não importando a localização dos mesmos na cavidade da boca, desde que as reabilitações estivessem adequadas. Aqueles indivíduos que foram identificados como portadores de toro mandibular bilateral foram incluídos como Grupo Casos e os que não apresentavam toro como Grupo Controles.

\section{Critérios de exclusão}

- Presença de outros distúrbios de origem sistêmica ou local, que não fosse a presença do toro mandibular e que poderiam comprometer o crescimento craniofacial ou sistema mastigatório, como distúrbios neurológicos, paralisia cerebral, entre outros; 
- Utilização de medicamentos que poderiam interferir na atividade muscular, direta ou indiretamente, como anti-histamínicos, sedativos, xaropes, homeopatia ou outras drogas depressoras do Sistema Nervoso Central;

- Realização de tratamentos que poderiam interferir na atividade muscular, direta ou indiretamente, durante o período da realização da pesquisa, como terapia fonoaudiológica e tratamento otorrinolaringológico.

\section{Material utilizado}

Foram utilizados durante a avaliação eletromiográfica da mastigação amendoins, uvas passas e Parafilme $\mathbf{M}^{\circledR}$ (parafina dobrada). A escolha desses materiais ocorreu por sua consistência similar com os alimentos duros (amendoins) e macios (uvas passas) utilizados diariamente pela população e o Parafilme $\mathbf{M}^{\circledR}$ por ser um material utilizado para execução de mastigação não-habitual padrão na literatura (PEPATO et al., 2013).

\section{Considerações Éticas}

Todos os indivíduos foram informados sobre os propósitos e etapas da pesquisa e assinaram o Termo de Consentimento Livre e Esclarecido, previamente aprovado pelo Comitê de Ética da Universidade Estadual de Montes Claros de acordo com a resolução 196/96 do Conselho Nacional de Saúde sob o parecer nº 226.704 de 22 de fevereiro de 2013 (Anexo I).

\section{AVALIAÇÃO ELETROMIOGRÁFICA}

Para o registro eletromiográfico nas condições posturais da mandíbula e na mastigação foram utilizados cinco canais do eletromiógrafo Myosystem - BR1 - DataHomins Ltda., com aquisição simultânea, aterramento comum a todos canais, filtros de baixa passagem de $10 \mathrm{~Hz}$ a $5 \mathrm{KHz}$; impedância de entrada dos canais de $10 \mathrm{G} \Omega$ em modo diferencial, 12 bites de faixa 
de resolução dinâmica, faixa de amplitude $-10 \mathrm{~V}$ a $+10 \mathrm{~V}$ e frequência de amostragem por canal de 2KHz (Figura 2).

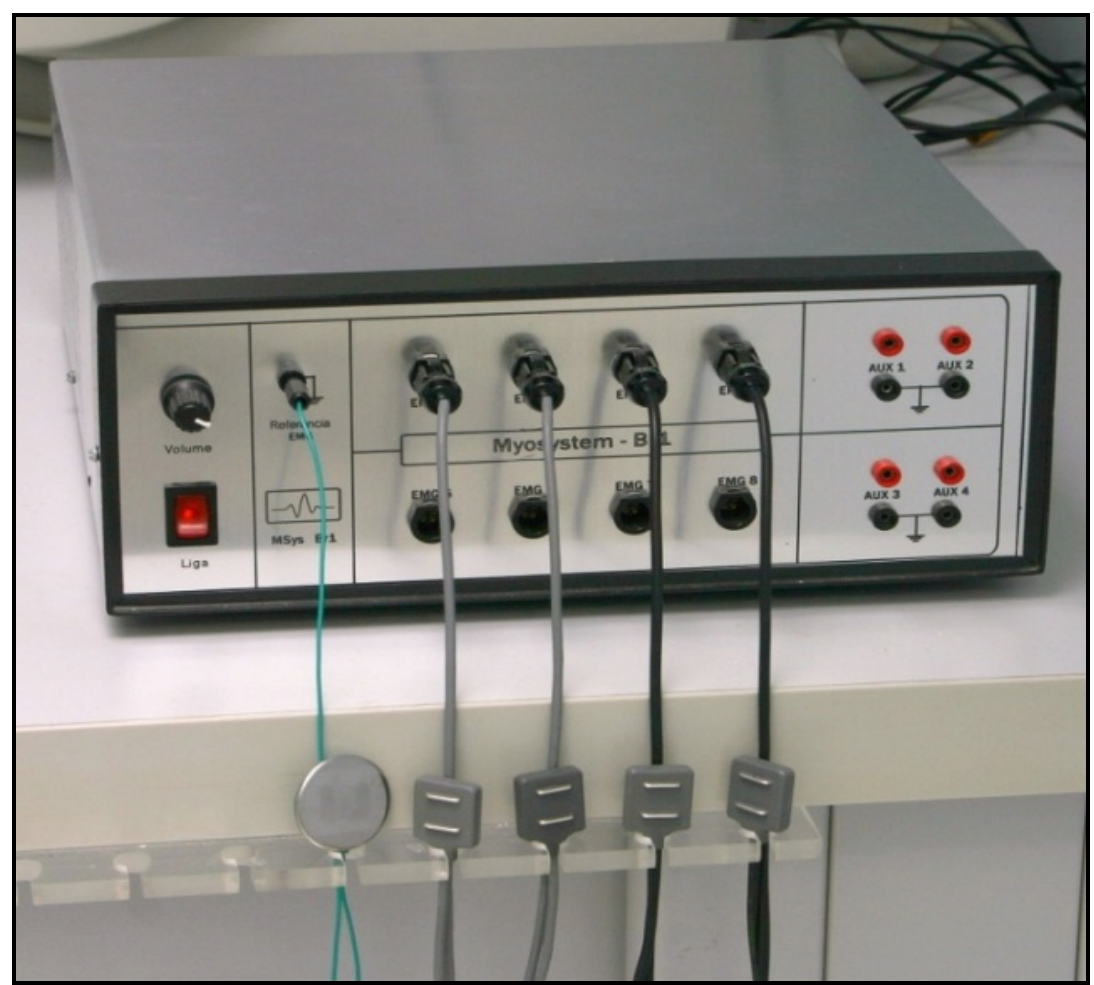

Figura 2. Myosystem - BR1 - DataHomins Tecnologia Ltda.

Para visualização e processamento dos sinais eletromiográficos foi utilizado o programa Myosystem I versão 3.5 que também permitiu, após a digitalização, que os sinais fossem analogicamente amplificados com um ganho de 1000x, filtrados por um filtro passabanda de 0,01-1,5kHz e amostrados por uma placa conversora A/D de 12 bites com freqüência de aquisição de 2 kHz.

Eletrodos ativos diferenciais de superfície (duas barras de cloreto de prata, de 10mm de comprimento por $2 \mathrm{~mm}$ de largura, afastadas em $10 \mathrm{~mm}$, com impedância de entrada de $10 \mathrm{G} \Omega$ e taxa de rejeição do modo comum de $130 \mathrm{~dB}$ a $60 \mathrm{~Hz}$ ) foram usados neste estudo (Figura 3). 


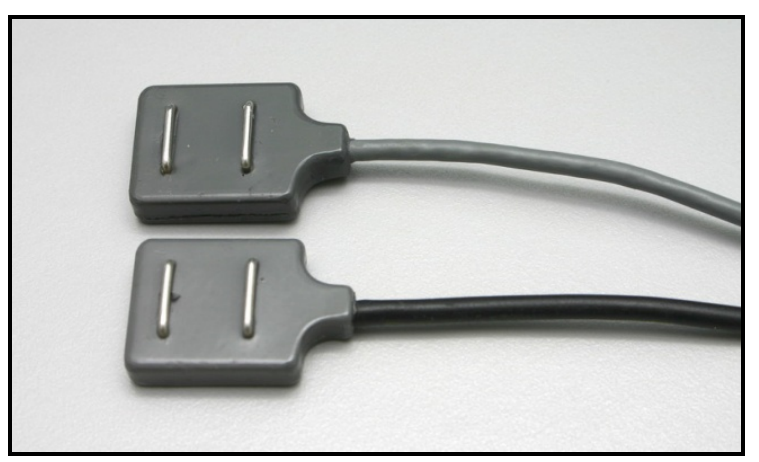

Figura 3. Eletrodo ativo de superfície

A pele da região de colocação dos eletrodos utilizada para avaliar os músculos masseter e temporal foi higienizada com álcool assim como tricotomizada quando necessária. A posição dos eletrodos foi determinada seguindo as recomendações de Cram et al. (1998), fixados por esparadrapos de tal forma que a maior extensão das barras ocupava o sentido perpendicular em direção das fibras musculares, ou seja, ventre muscular dos músculos masseteres direito (MD) e esquerdo (ME), temporal direito (TD) e esquerdo (TE).

Um eletrodo circular de aço inoxidável (três centímetros de diâmetro) também foi usado como eletrodo de referência (eletrodo terra), fixado sobre a pele da região do pulso ou punho (Figura 4).

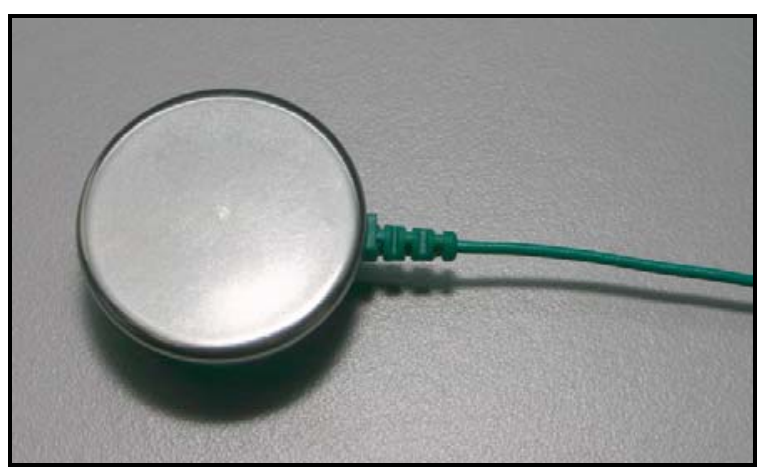

Figura 4. Eletrodo circular de aço inoxidável (referência terra) 
Eletromiografia nas condições posturais da mandíbula

A avaliação da atividade muscular foi realizada por meio de registros eletromiográficos da musculatura avaliada durante o repouso, no MIH e no apertamento dental máximo com Parafilme $\mathbf{M}^{\circledR}$ entre os dentes molares (por quatro segundos), assim como nas seguintes condições clínicas: lateralidade esquerda e direita máximas com contato dental e protrusão máxima da mandíbula com contato dental, todas durante 10 segundos.

\section{Eletromiografia nas condições de mastigação habitual e não-habitual}

A eficiência do ciclo mastigatório foi analisada por meio da integral da envoltória do sinal eletromiográfico obtido na mastigação habitual de alimento duro (3g de amendoim) e de alimento macio (3g de uvas passas), durante dez segundos. Também foram adquiridos sinais eletromiográficos na condição clínica durante a mastigação não-habitual contínua de um material inerte durante dez segundos. Este material inerte constitui-se em uma folha de parafina (Parafilme $\mathrm{M}^{\circledR}$ ) que foi dobrada (18x17x4mm, peso $245 \mathrm{mg}$ ) e colocada em ambos os lados da arcada dental (Figura 5).
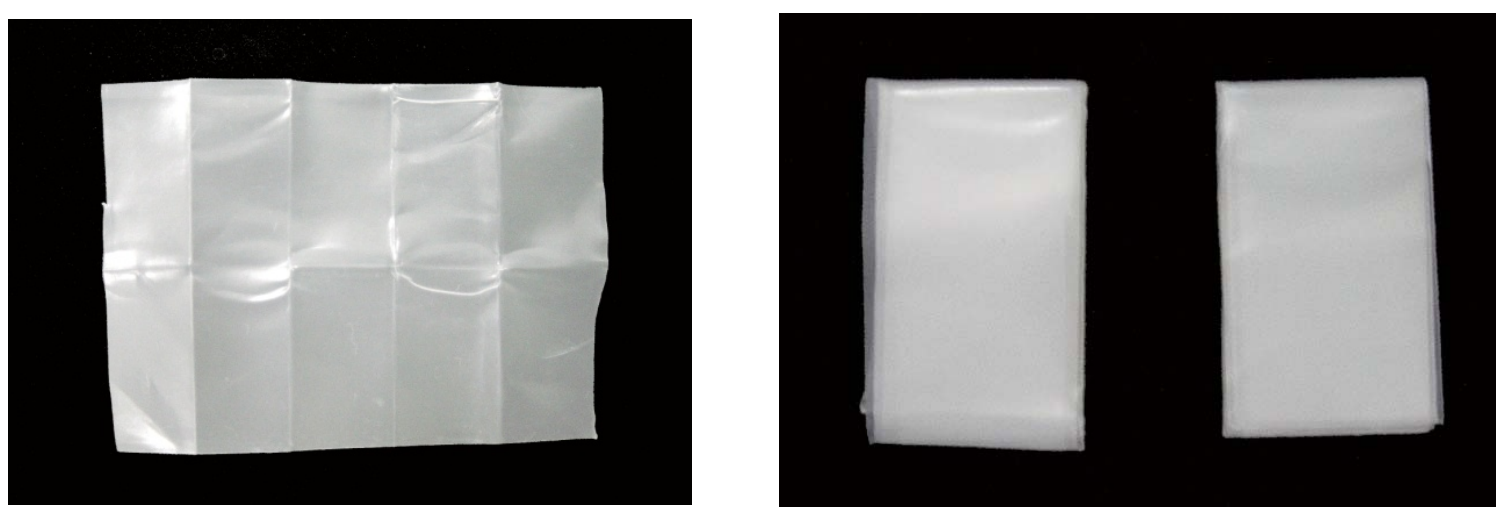

Figura 5. Material inerte - folha de parafina (Parafilme $\left.M^{\circledR}\right)$ que foi dobrada $(18 \times 17 x 4 m m$, peso $245 \mathrm{mg})$. 


\section{ANÁLISE ESTATÍSITCA}

Os sinais eletromiográficos foram processados no programa Myosystem - Br1 versão 3.5. Após a digitalização, os sinais foram analogicamente amplificados (com um ganho de 1000x), filtrados (filtro passa-banda de $0,01-1,5 \mathrm{kHz}$ ) e amostrados por uma placa conversora A/D de 12 bites com frequência de aquisição de $2 \mathrm{KHz}$. O sinal eletromiográfico bruto foi utilizado para derivar valores de amplitude eletromiográfica, obtidos pelo cálculo da raiz quadrada da média (RMS).

Os dados eletromiográficos das condições posturais da mandíbula foram tabulados e submetidos à análise estatística utilizando o software SPSS versão 19.0 para Windows (SPSS Inc.; Chicago, IL, USA). Foi realizada a análise descritiva (médias, desvios padrão, valor máximo e valor mínimo), para cada variável. Os valores obtidos foram comparados pelo teste $t$ para amostras independentes $(\mathrm{p}<0,05)$. A eficiência do ciclo mastigatório entre os grupos foi verificada por meio da integral da envoltória do sinal eletromiográfico, sendo este valor em microvolts/segundo. 
RESULTADOS 



\section{RESULTADOS}

Participaram desta pesquisa 40 indivíduos adultos com idade média de 28,23 $( \pm 10,54)$ anos, sendo 24 do gênero feminino (60\%) e 16 do gênero masculino (40\%), divididos em dois Grupos de 20 indivíduos cada, pareados sujeito a sujeito: Grupo Casos, com indivíduos portadores de toro mandibular bilateral e Grupo Controles com indivíduos sem toro mandibular.

\section{RESULTADOS ELETROMIOGRÁFICOS PARA AS CONDIÇÕES CLÍNICAS POSTURAIS DA MANDÍBULA}

\section{Condição Clínica de Repouso}

Para a condição clínica de Repouso, os indivíduos do Grupo Casos apresentaram uma maior atividade eletromiográfica dos músculos masseteres (D e E), enquanto os indivíduos do Grupo Controles apresentaram uma maior atividade eletromiográfica dos músculos temporais (D e E) (Figura 6). Entretanto, essas diferenças não foram estatisticamente significantes (Tabela 1). 


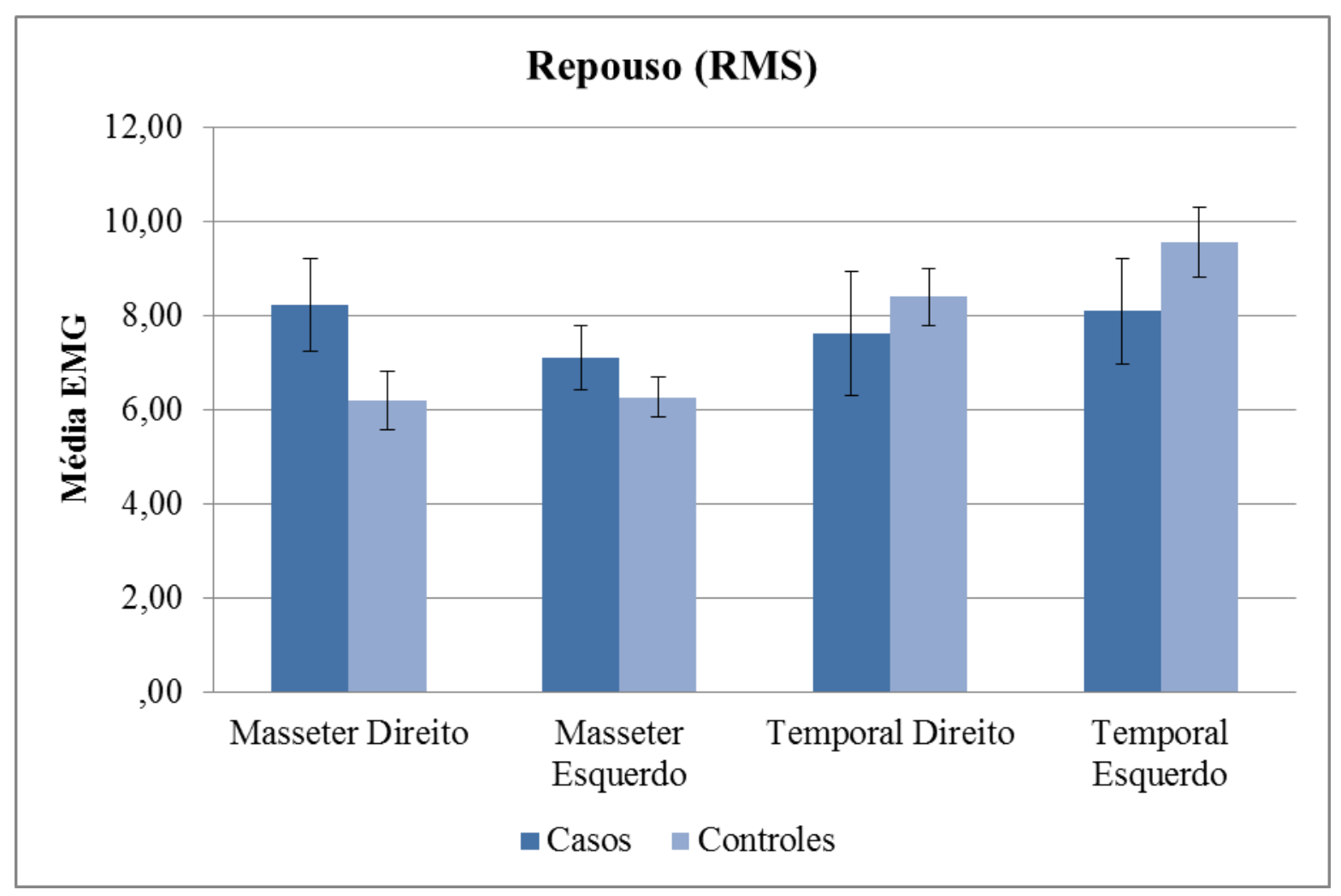

Figura 6. Média e erro padrão dos valores eletromiográficos (RMS) dos músculos masseter e temporal na condição clínica de Repouso para os dois grupos analisados: Casos X Controles.

Tabela 1 - Teste t das médias eletromiográficas entre os dois grupos analisados: Casos X Controles. Dados em RMS, condição clínica Repouso ( $<<0,05)$.

\begin{tabular}{|lccc}
\hline \multicolumn{1}{c}{ Músculo } & Casos & Controles & P \\
& $\dot{\mathbf{x}}( \pm)$ & $\dot{\mathbf{x}}( \pm)$ & \\
\hline Masseter Direito & $8,22( \pm 0,98)$ & $6,19( \pm 0,62)$ & 0,087 \\
\hline Masseter Esquerdo & $7,11( \pm 0,68)$ & $6,26( \pm 0,43)$ & 0,301 \\
\hline Temporal Direito & $7,62( \pm 1,33)$ & $8,39( \pm 0,62)$ & 0,602 \\
\hline Temporal Esquerdo & $8,10( \pm 1,12)$ & $9,55( \pm 0,75)$ & 0,288 \\
\hline
\end{tabular}




\section{CONDIÇÃO CLÍNICA: PROTRUSÃO}

Na condição clínica de Protrusão, os indivíduos do Grupo Casos apresentaram uma maior atividade eletromiográfica dos músculos masseteres (D e E) estatisticamente significante em relação aos indivíduos do Grupo Controles $(\mathrm{p}<0,05)$ (Tabela 2).

Assim como na condição clínica de Repouso, na condição clínica de Protrusão, evidenciou-se que para o músculo temporal direito, os indivíduos do Grupo Controles também apresentaram uma maior atividade eletromiográfica em relação ao Grupo Casos. Entretanto, essa diferença não foi estatisticamente significante (Figura 7 e Tabela 2). Observou-se, ainda nesse movimento, maior atividade eletromiográfica dos músculos masseteres quando comparada aos temporais em ambos os lados, para os dois grupos analisados. Observou-se que os dois grupos mantiveram o padrão de ativação esperado para a execução da condição clínica de Protrusão, com os masseteres mais ativos que os temporais, destacando um maior equilíbrio entre os lados para o Grupo Controles (Figura 7). 


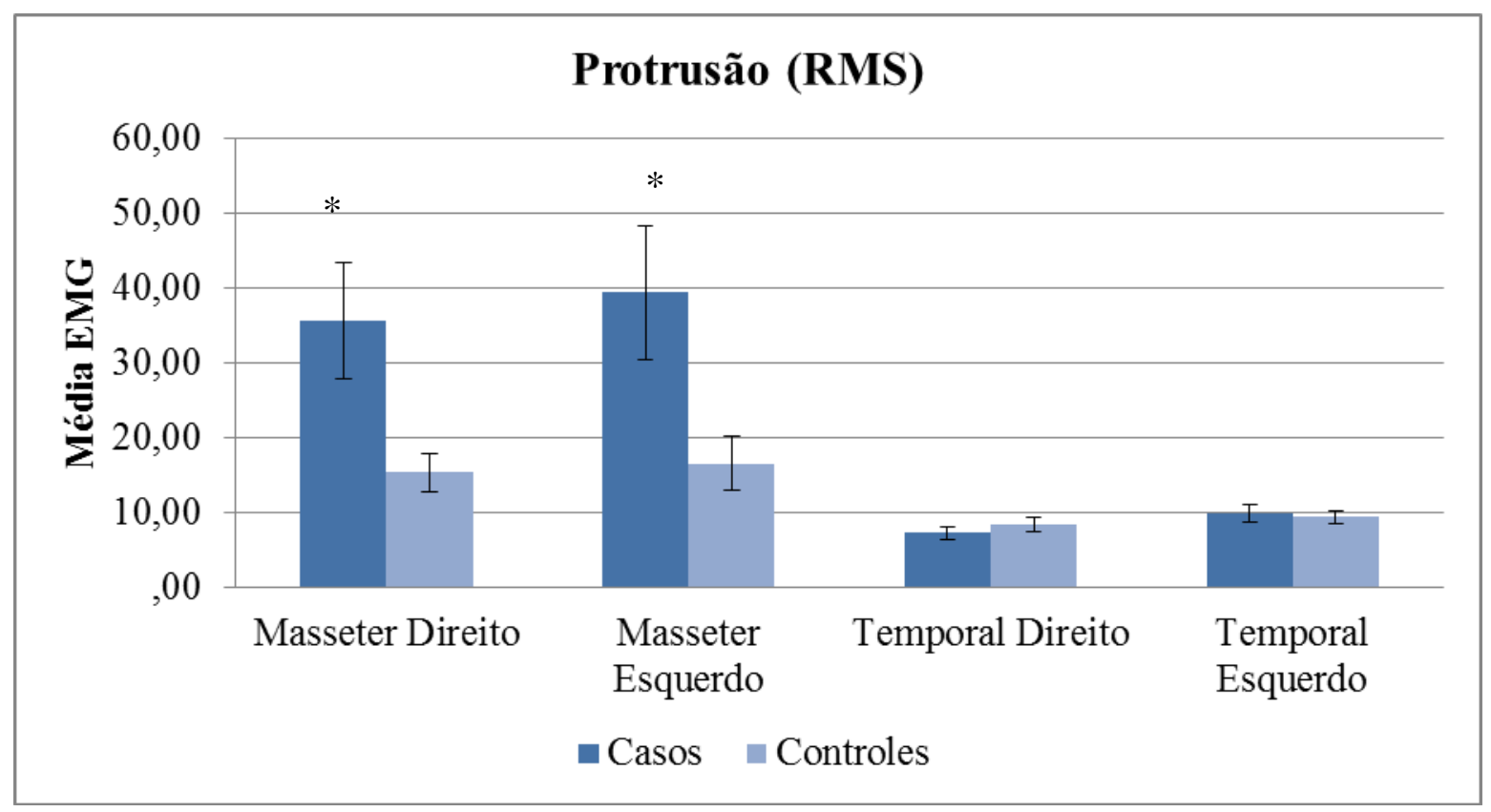

Figura 7. Média e erro padrão dos valores eletromiográficos (RMS) dos músculos masseter e temporal na condição clínica de Protrusão para os dois grupos analisados: Casos X Controles

Tabela 2 - Teste t das médias eletromiográficas entre os dois grupos analisados: Casos X Controles. Dados em RMS, condição clínica Protrusão ( $<<0,05)$.

\begin{tabular}{lccc}
\hline \multicolumn{1}{c}{ Músculo } & Casos & Controles & \\
& $\dot{\mathrm{X}}( \pm)$ & $\dot{\mathrm{x}}( \pm)$ & $\mathbf{p}$ \\
\hline Masseter Direito & $35,64( \pm 7,84)$ & $15,28( \pm 2,59)$ & $0,022^{*}$ \\
\hline Masseter Esquerdo & $39,35( \pm 8,92)$ & $16,53( \pm 3,53)$ & $0,025^{*}$ \\
\hline Temporal Direito & $7,22( \pm 0,83)$ & $8,31( \pm 0,89)$ & 0,372 \\
\hline Temporal Esquerdo & $9,73( \pm 1,16)$ & $9,29( \pm 0,86)$ & 0,763 \\
\hline * Significante $(\mathrm{p}<0,05)$. & & & \\
\hline
\end{tabular}




\section{CONDIÇÃO CLÍNICA: APERTAMENTO DENTAL}

A condição clínica de Apertamento foi avaliada de duas formas: Máxima Intercuspidação Habitual (MIH) e Apertamento Dental com Parafilme $\mathrm{M}^{\circledR}$ (AP). Na condição clínica de apertamento dental, em ambas as formas avaliadas, os indivíduos do Grupo Casos apresentaram maior atividade eletromiográfica dos músculos masseteres (D e E) estatisticamente significante em relação aos indivíduos do Grupo Controles (p<0,01) (Tabela 3).

Nos músculos temporais (D e E) não houve diferença estatisticamente significante entre os grupos (Tabela 3). Observou-se, ainda, maior atividade eletromiográfica dos músculos masseteres quando comparada aos temporais em ambos os lados, tanto na $\mathrm{MIH}$, quanto no AP, para o Grupo Casos (Figuras 8 e 9). Na MIH e no Apertamento Dental com Parafilme $\mathrm{M}^{\circledR}$ (AP) verificou-se atividade eletromiográfica similar para todos os músculos avaliados, em ambos os músculos para o Grupo Controles (Figuras 8 e 9).

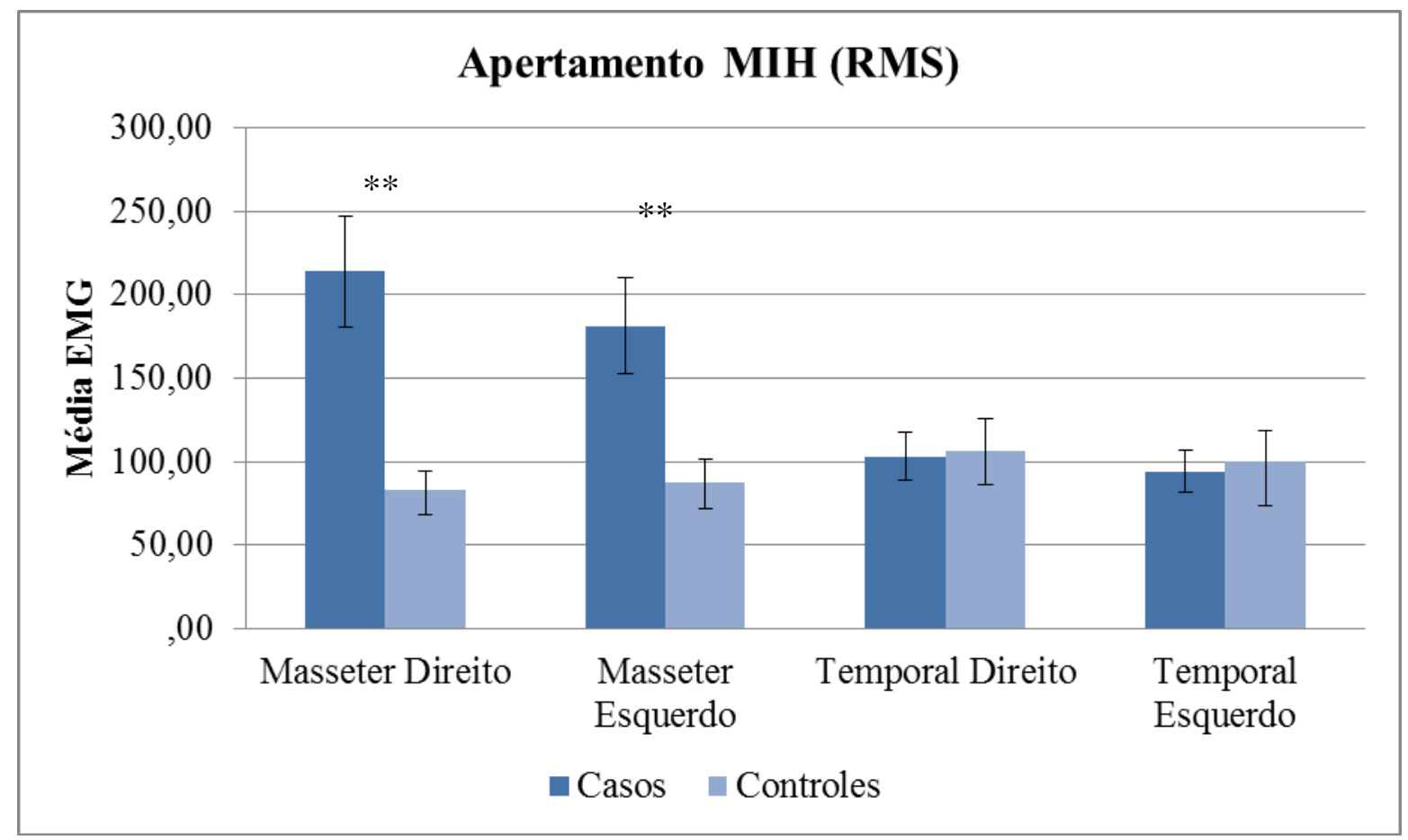

Figura 8. Média e erro padrão dos valores eletromiográficos (RMS) dos músculos masseter e temporal na condição clínica de Máxima Intercuspidação Habitual (MIH) para os dois grupos analisados: Casos X Controles. 


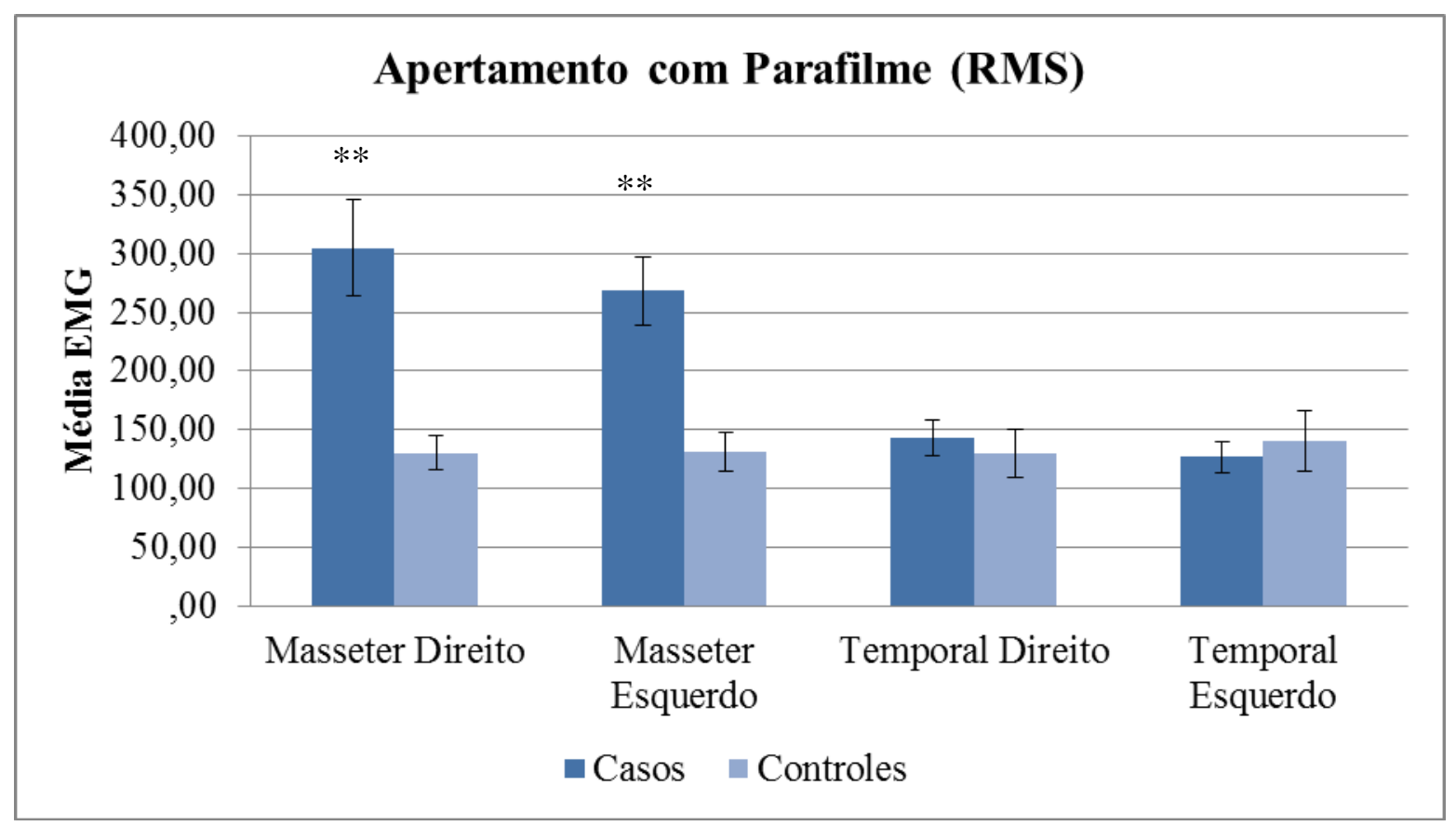

Figura 9. Média e erro padrão dos valores eletromiográficos (RMS) dos músculos masseter e temporal na condição clínica de Apertamento Dental com Parafilme $\mathbf{M}^{\circledR}$ para os dois grupos analisados: Casos X Controles.

Tabela 3 - Teste t das médias eletromiográficas entre os dois grupos analisados: Casos X Controles. Dados em RMS, condição clínica Apertamento na Máxima Intercuspidação Habitual (MIH) e Apertamento Dental com Parafilme $\mathbf{M}^{\circledR}(\mathrm{p}<0,05)$.

\begin{tabular}{|c|c|c|c|}
\hline Músculo & $\begin{array}{l}\text { Casos } \\
\dot{\mathrm{x}}( \pm)\end{array}$ & $\begin{array}{l}\text { Controles } \\
\dot{x}( \pm)\end{array}$ & $\mathbf{p}$ \\
\hline \multicolumn{4}{|l|}{ Apertamento (MIH) } \\
\hline Masseter Direito & $213,78( \pm 33,47)$ & $82,92( \pm 11,64)$ & $0,001 * *$ \\
\hline Masseter Esquerdo & $181,33( \pm 28,99)$ & $87,67( \pm 13,68)$ & $0,007 * *$ \\
\hline Temporal Direito & $103,29( \pm 14,34)$ & $106,78( \pm 18,57)$ & 0,883 \\
\hline Temporal Esquerdo & $94,28( \pm 12,55)$ & $99,76( \pm 18,57)$ & 0,808 \\
\hline \multicolumn{4}{|c|}{ Apertamento (Parafilme) } \\
\hline Masseter Direito & $304,53( \pm 40,85)$ & $130,23( \pm 14,69)$ & $0,001^{* *}$ \\
\hline Masseter Esquerdo & $268,21( \pm 29,28)$ & $131,13( \pm 16,05)$ & $0,000 * *$ \\
\hline Temporal Direito & $143,20( \pm 15,68)$ & $129,62( \pm 20,61)$ & 0,603 \\
\hline Temporal Esquerdo & $126,90( \pm 12,98)$ & $140,64( \pm 25,66)$ & 0,637 \\
\hline
\end{tabular}




\section{CONDIÇÃO CLÍNICA: LATERALIDADE}

Durante os movimentos de lateralidade houve significância estatística entre os grupos para o músculo masseter, enquanto para o temporal não ocorreu. Na lateralidade direita houve diferença significativa na atividade eletromiográfica entre os grupos para o músculo masseter esquerdo $(\mathrm{p}<0,01)$. Já na lateralidade esquerda houve diferença significativa entre os grupos, para o músculo masseter direito $(\mathrm{p}<0,05)$ (Tabela 4).

Observou-se, ainda, que na lateralidade direita houve maior atividade eletromiográfica dos músculos masseter esquerdo e temporal direito, para ambos os grupos. Sendo que, o Grupo Casos apresentou maior atividade eletromiográfica no músculo masseter, enquanto o Grupo Controles apresentaram maior atividade eletromiográfica no músculo temporal (Figura 10).

$\mathrm{Na}$ lateralidade esquerda, houve maior atividade eletromiográfica dos músculos masseter direito e temporal esquerdo, para o Grupo Controles, destacando a atividade do músculo temporal em relação ao masseter. Para o Grupo Casos, na lateralidade esquerda, houve maior atividade eletromiográfica dos músculos masseter direito, masseter esquerdo e temporal esquerdo, respectivamente nessa ordem, o que demonstrou maior atividade eletromiográfica do músculo masseter em relação ao temporal, para o referido grupo (Figura 11). 


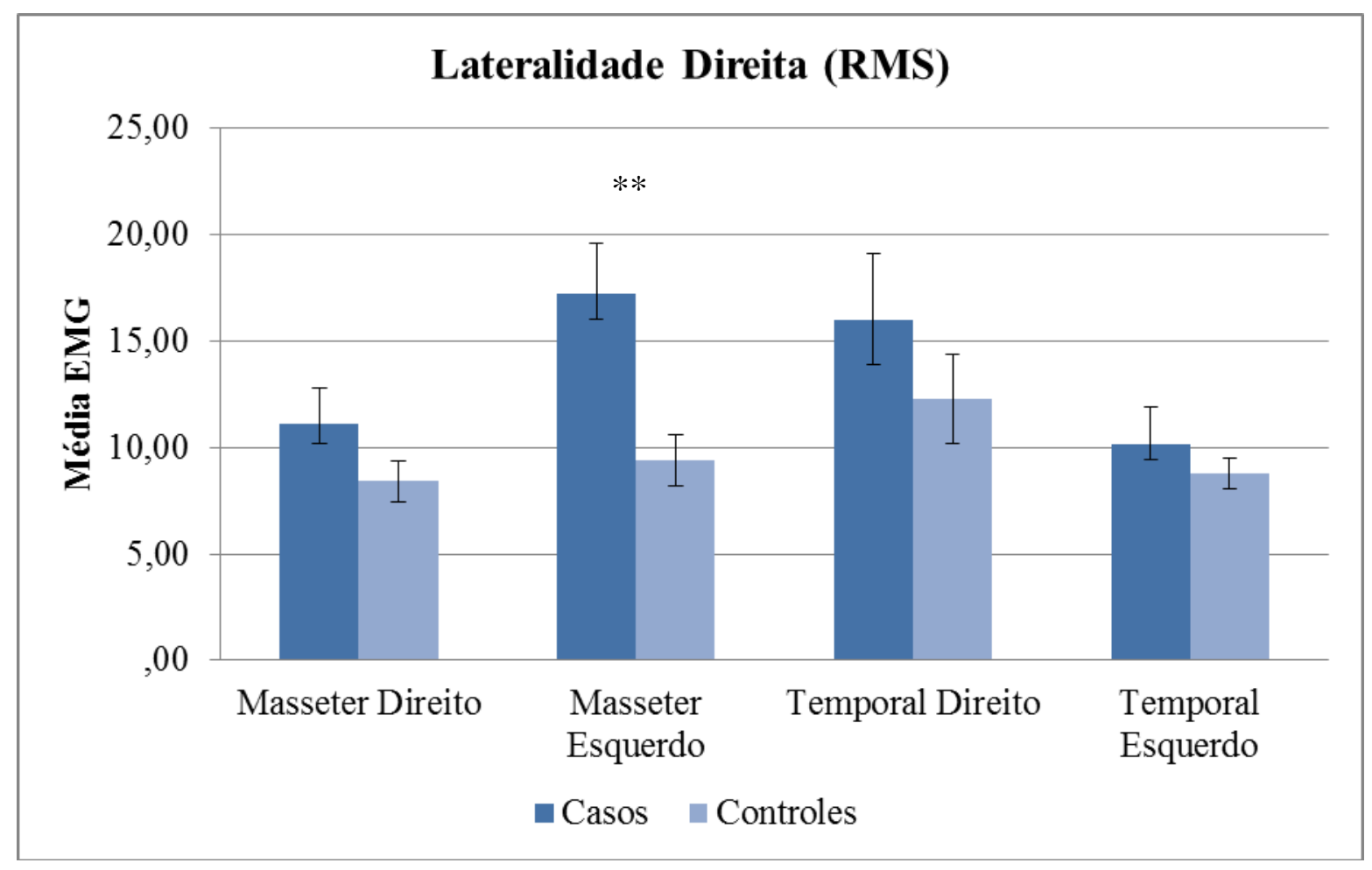

Figura 10. Média e erro padrão dos valores eletromiográficos (RMS) dos músculos masseter e temporal na condição clínica de lateralidade direita para os dois grupos analisados: Casos X Controles.

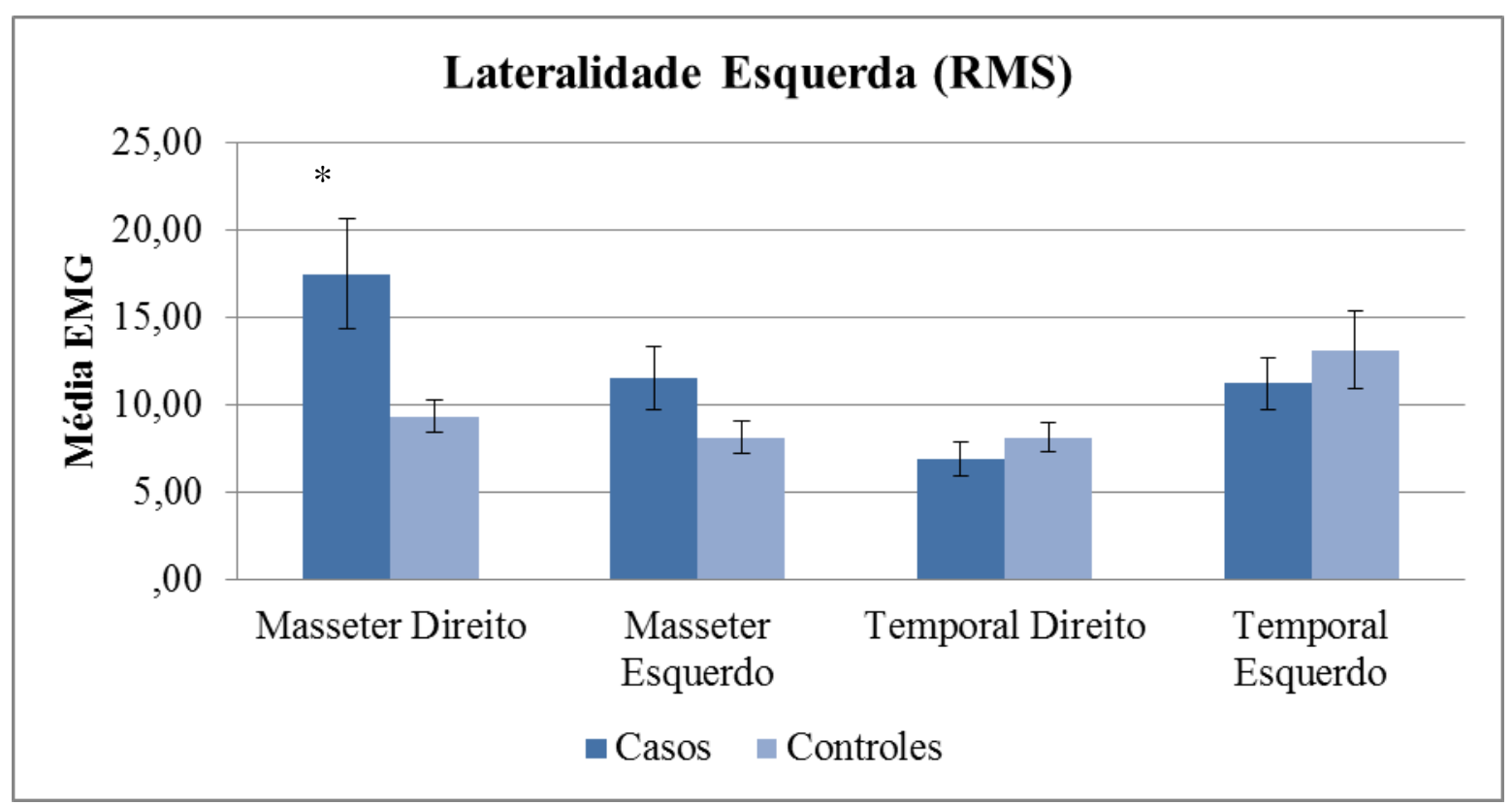

Figura 11. Média e erro padrão dos valores eletromiográficos (RMS) dos músculos masseter e temporal na condição clínica de lateralidade esquerda para os dois grupos analisados: Casos $\mathrm{X}$ Controles. 
Tabela 4 - Teste t das médias eletromiográficas entre os dois grupos analisados: Casos $\mathrm{X}$ Controles. Dados em RMS, condição clínica Lateralidades Direita e Esquerda $(p<0,05)$.

\begin{tabular}{|cccc}
\hline \multicolumn{1}{c}{ Músculo } & Casos & Controles & \\
& $\dot{\mathbf{X}}( \pm)$ & $\dot{\mathbf{X}}( \pm)$ & $\mathbf{p}$ \\
\hline Lateralidade Direita & & & \\
\hline Masseter Direito & $11,12( \pm 1,69)$ & $8,41( \pm 0,97)$ & 0,172 \\
\hline Masseter Esquerdo & $17,22( \pm 2,38)$ & $9,40( \pm 1,18)$ & $0,006^{* *}$ \\
\hline Temporal Direito & $16,01( \pm 3,10)$ & $12,29( \pm 2,09)$ & 0,327 \\
\hline Temporal Esquerdo & $10,13( \pm 1,77)$ & $8,76( \pm 0,70)$ & 0,480 \\
\hline Lateralidade Esquerda & & & \\
\hline Masseter Direito & $17,45( \pm 3,15)$ & $9,30( \pm 0,91)$ & $0,021^{*}$ \\
\hline Masseter Esquerdo & $11,49( \pm 1,84)$ & $8,11( \pm 0,92)$ & 0,108 \\
\hline Temporal Direito & $6,88( \pm 0,98)$ & $8,10( \pm 0,82)$ & 0,346 \\
\hline Temporal Esquerdo & $11,22( \pm 1,48)$ & $13,12( \pm 2,21)$ & 0,477 \\
\hline
\end{tabular}

* Significante $(\mathrm{p}<0,05)$

** Significante $(\mathrm{p}<0,01)$ 
RESULTADOS ELETROMIOGRÁFICOS PARA AS CONDIÇÕES CLÍNICAS DE MASTIGAÇÃO

A condição clínica de mastigação é uma atividade dinâmica, portanto para essa condição foram utilizados dados da envoltória linear em lugar da RMS. A mastigação foi avaliada de três formas: utilizando Parafilme $\mathbf{M}^{\circledR}$, amendoins e uvas passas.

Nas três formas de avaliação feitas para a condição clínica de mastigação, os indivíduos do Grupo Casos apresentaram atividade eletromiográfica de todos os músculos avaliados significativamente maior em relação aos indivíduos do Grupo Controles ( $\mathrm{p}<0,01)$ (Tabela 5).

Em todas as formas de avaliação da mastigação, o Grupo Casos apresentaram maior atividade eletromiográfica do músculo masseter em relação ao temporal, enquanto o Grupo Controles apresentaram atividade eletromiográfica homogênea entre os músculos avaliados (Figuras 12, 13 e 14).

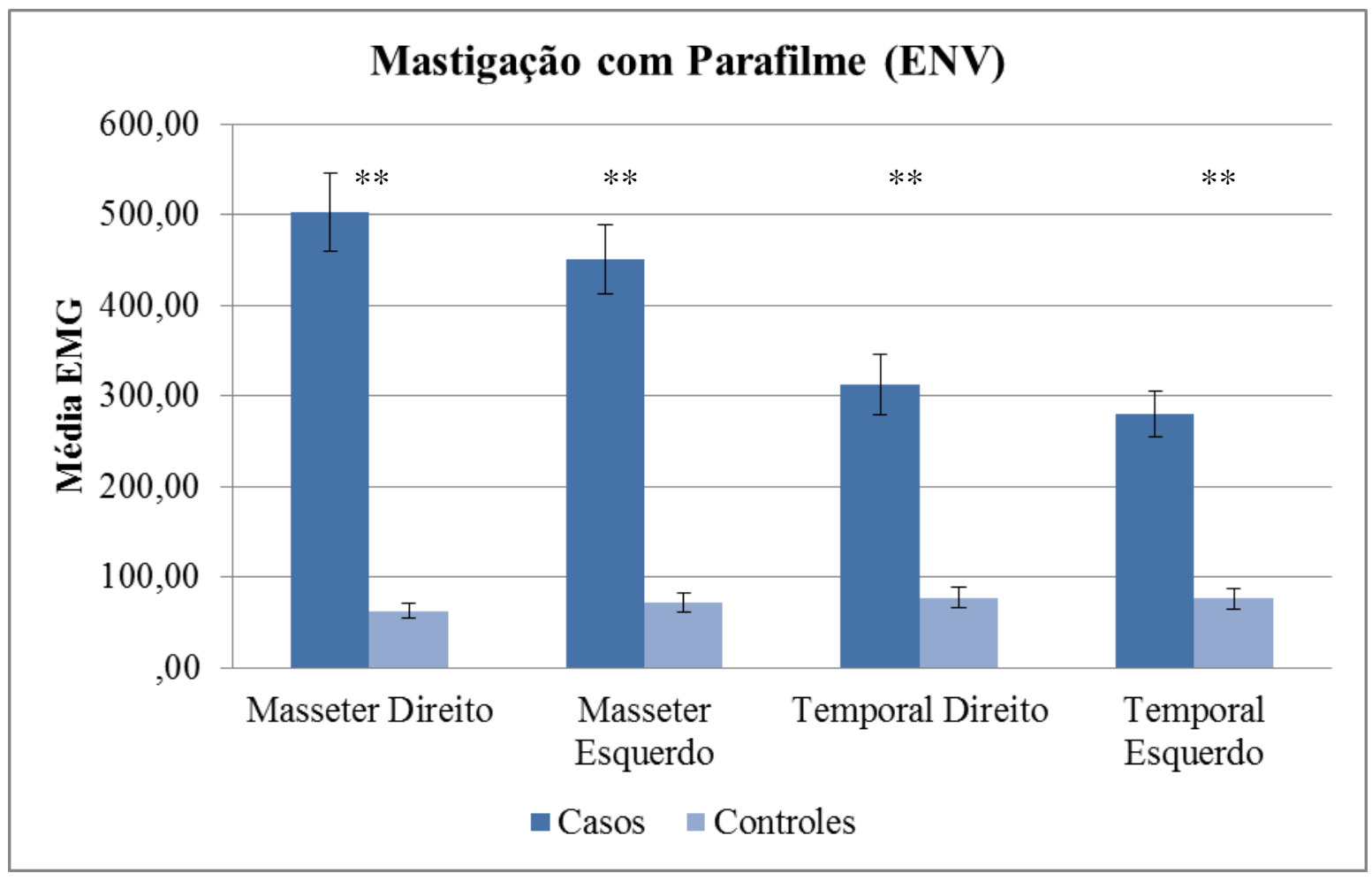

Figura 12. Média e erro padrão dos valores eletromiográficos (ENV) dos músculos masseter e temporal na condição clínica de mastigação com parafilme para os dois grupos analisados: Casos X Controles. 


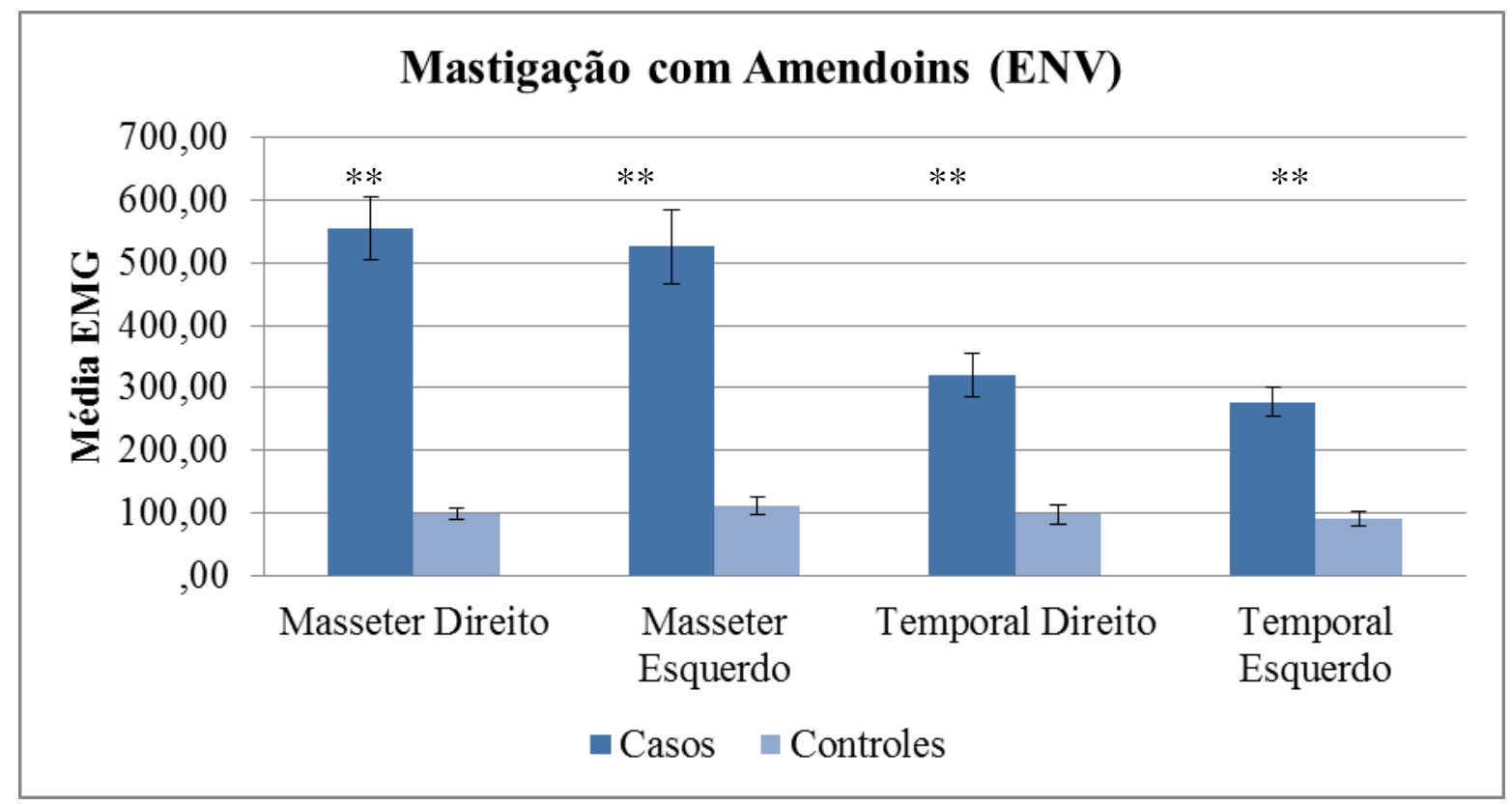

Figura 13. Média e erro padrão dos valores eletromiográficos (ENV) dos músculos masseter e temporal na condição clínica de mastigação amendoins para os dois grupos analisados: Casos X Controles.

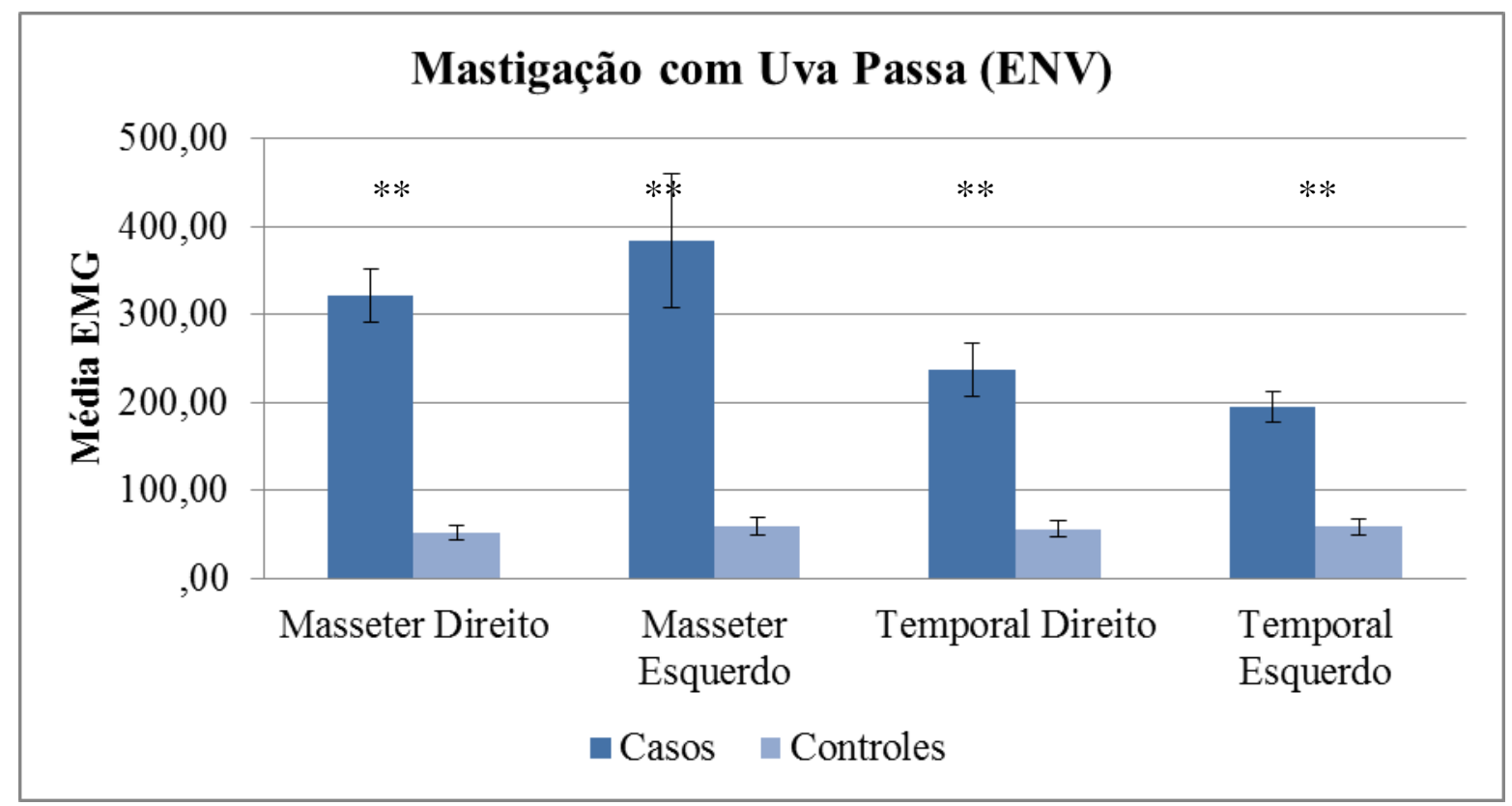

Figura 14. Média e erro padrão dos valores eletromiográficos (ENV) dos músculos masseter e temporal na condição clínica de mastigação com uva passas para os dois grupos analisados: Casos X Controles. 
Tabela 5 - Teste "t" de comparação das médias dos valores eletromiográficos (RMS) dos músculos masseter e temporal nas condições clínicas de do Grupo Casos X Grupo Controles.

\begin{tabular}{|c|c|c|c|}
\hline \multirow[b]{2}{*}{ Músculo } & Casos & Controles & \multirow[b]{2}{*}{$\mathbf{p}$} \\
\hline & & & \\
\hline \multicolumn{4}{|c|}{ Mastigação com Parafilme } \\
\hline Masseter Direito & $503,02( \pm 43,06)$ & $63,03( \pm 7,68)$ & $0,000 * *$ \\
\hline Masseter Esquerdo & $450,58( \pm 38,21)$ & $72,17( \pm 9,91)$ & $0,000 * *$ \\
\hline Temporal Direito & $313,12( \pm 33,55)$ & $77,54( \pm 11,58)$ & $0,000 * *$ \\
\hline Temporal Esquerdo & $280,02( \pm 25,63)$ & $76,81( \pm 11,30)$ & $0,000 * *$ \\
\hline \multicolumn{4}{|c|}{ Mastigação com Amendoins } \\
\hline Masseter Direito & $553,90( \pm 50,11)$ & $98,97( \pm 10,20)$ & $0,000 * *$ \\
\hline Masseter Esquerdo & $525,21( \pm 58,35)$ & $112,31( \pm 13,90)$ & $0,000 * *$ \\
\hline Temporal Direito & $320,14( \pm 35,62)$ & $98,49( \pm 15,36)$ & $0,000 * *$ \\
\hline Temporal Esquerdo & $277,60( \pm 23,84)$ & $91,42( \pm 11,09)$ & $0,000 * *$ \\
\hline \multicolumn{4}{|c|}{ Mastigação com Uvas Passas } \\
\hline Masseter Direito & $321,51( \pm 29,54)$ & $52,29( \pm 0,08)$ & $0,000 * *$ \\
\hline Masseter Esquerdo & $383,58( \pm 76,38)$ & $59,01( \pm 9,85)$ & $0,000 * *$ \\
\hline Temporal Direito & $237,75( \pm 30,25)$ & $56,47( \pm 9,19)$ & $0,000 * *$ \\
\hline Temporal Esquerdo & $195,87( \pm 17,41)$ & $58,95( \pm 8,53)$ & $0,000 * *$ \\
\hline
\end{tabular}

** Significante $(\mathrm{p}<0,01)$. 
DISCUSSÃO 



\section{DISCUSSÃO}

O toro é considerado uma anomalia do desenvolvimento, com etiologia multifatorial, atribuída a fatores genéticos, ambientais, hiperfunção mastigatória e com crescimento lento durante toda a vida (SIRIRUNGROJYING; KERDPON, 1999; NASCIMENTO FILHO et al., 2004; BUKHARI et al., 2007).

O predomínio da presença do toro ocorre na faixa etária de adulto jovem e indivíduos de meia-idade (SIRIRUNGROJYING; KERDPON,1999; CANTO, 2009), fato esse verificado nesta amostra para os adultos jovens, onde a faixa etária dos indivíduos participantes da pesquisa foi 28,23 $( \pm 10,15)$. O toro mandibular é encontrado apenas na superfície lingual da mandíbula, na região dos pré-molares, com maior predomínio em homens do que em mulheres, dado esse semelhante aos encontrados nos nossos resultados, onde o maior predomínio dos toros foi em homens (FURTADO et al., 2008).

O toro geralmente não tem indicação clínica de remoção e nem de tratamento, a menos que se torne tão grande que possa interferir com a função, principalmente durante a fala ou ainda com a instalação de próteses reabilitadoras. Recentemente alguns trabalhos sugeriram uma relação da presença de toro mandibular com bruxismo ou com disfunção temporomandibular (DTM), fatores esses que foram critérios de exclusão dos participantes desse estudo, para não influenciarem nos resultados obtidos. Nenhuns dos participantes tinham diagnóstico de bruxônamos ou apresentavam sinais e sintomas de disfunção temporomandibular, avaliados pelo Índice Clínico de Fonseca e o RDC/TMD. (SIRIRUNGROJYING, KERDPON, 1999; SERRA, CAMPOS, 2005; SERRA et al., 2005; IGARASHI et al., 2008; FURTADO et al., 2008; SHANKLAND, 2010).

Para analisar as implicações de desordens funcionais na musculatura pode-se utilizar a eletromiografia de superfície (EMG). Na Odontologia, as pesquisas com eletromiografia foram 
iniciadas em 1949, com Moyers, na análise do comportamento da musculatura do sistema estomatognático frente a diferentes desordens oclusais e tipos faciais. Atualmente, a análise eletromiográfica representa um meio de diagnóstico, assim como de avaliação e acompanhamento de tratamentos realizados (REGALO et al., 2003; SANTOS et al., 2008, SIÉSSERE et al., 2009), por meio do registro dos potenciais de ação das fibras musculares e análise da coordenação dos músculos do sistema estomatognático (JANKELSON, 1990; MARCHIORI; VITTI,1996; COOPER, 1997). Trata-se de um método seguro e eficaz para a avaliação da atividade muscular, podendo ser utilizado tanto em adultos quanto em crianças (FERRARIO et al., 2002).

Jankelson (1990) descreveu que monitorar o nível da atividade muscular em repouso por meio da eletromiografia é uma técnica eficiente para verificar a condição fisiológica do sistema estomatognático, afirmando que em caso de hipertonicidade muscular, provavelmente o indivíduo é portador de algum tipo de disfunção. Segundo Liu et al. (1999) e Pinho et al. (2000), a atividade eletromiográfica dos músculos da mastigação em repouso é maior nos indivíduos com disfunções do sistema estomatognático quando comparada com indivíduos saudáveis, o que indica um aumento do tônus basal da musculatura. A condição clínica de repouso mandibular é aquela na qual a mandíbula está estável, imóvel, sustentada por coordenação recíproca dos músculos da mastigação e músculos abaixadores da mandíbula, onde estes músculos se apresentam na condição de tônus neuromuscular, um estado de resistência passiva ao estiramento das fibras, mantendo a mandíbula em posição antigravitacional (HOUSSAY, 1966), com “silêncio eletromiográfico” característico desta posição relaxada (SGOBBI DE FARIA; BÉRZIN, 1998; VOUDOURIS; KUFTINIC, 2000; REGALO et al., 2006b). Nos nossos resultados foi possível observar que na condição clínica de repouso todos os indivíduos apresentaram atividade eletromiográfica para todos os músculos avaliados, sendo a atividade maior para os músculos masseteres do Grupo Casos, o que já sinalizou que a presença do toro mandibular pode estar estimulando a ativação das 
fibras destes músculos, mesmo no repouso, resultados estes, discordes da literatura, que evidenciou que os músculos em repouso não apresentam atividade elétrica, ou seja, não há contrações de unidades motoras (YEMM; NORDSTRÖM, 1974; THOMSON, 1981; CHONG et al.; 1991). Outros autores como Vitti e Basmajian, 1975; Basmajian e De Luca, 1985; Ferrario et al, 1993; Regalo et al., 2006a encontraram atividade elétrica mínima no repouso mandibular, resultados estes concordes com os verificados em nosso estudo para os dois grupos avaliados.

A condição clínica de repouso tem sido muito utilizada na Reabilitação Oral como um parâmetro clínico para análise oclusal em casos de próteses, implantes, cirurgias ortognáticas e desordens temporomandibulares (ARITA, 2000, BERSANI et al., 2011; Da SILVA et al., 2011; De ROSSI et al., 2013). O sistema estomatognático permanece a maior parte do tempo em repouso, com atividade em $12 \%$ durante o dia e no resto do período os músculos devem estar relaxados (TRENOUTH, 2000; CARR, 1991).

Qualquer alteração no equilíbrio do sistema estomatognático pode causar mudanças na tensão muscular (LIU et al., 1999; LANDULPHO et al., 2004) fato verificado nos resultados da presente investigação, em que foi possível observar que para as condições clínicas de protrusão, MIH, Apertamento Dental com Parafilme e lateralidade direita e esquerda, os músculos masseteres do Grupo Casos foram mais ativos que os do Grupo Controles, com dados estatisticamente significantes. O padrão de ativação muscular permaneceu, com os valores de atividade dos músculos masseteres predominando sobre os valores dos músculos temporais nas condições clínicas de Apertamento Dental, pelas próprias características morfológicas e funcionais destes músculos, com o músculo masseter mais potente, com função de força, que carrega e sustenta os ossos protegendo e conduzindo a potência do movimento e elevando a mandíbula antigravitacionalmente durante as diversas funções orais e o músculo temporal com função mais relacionada com a velocidade, sendo o primeiro a se 
contrair no fechamento mandibular, considerado um posicionador da mandíbula, ajusta melhor a direção do movimento, atuando como sincronizador de movimentos (VITTI; BASMAJIAN, 1975; HARPER et al., 1997; UEDA et al., 1998, ARITA, 2000; OKESON, 2000; SIMÕES, 2003; RANCAN et al., 2009; BERSANI et al., 2011; Da SILVA et al., 2011; De ROSSI et al., 2013).

Com relação às condições clínicas de protrusão e lateralidade (direita e esquerda), os indivíduos portadores de toro mandibular (Grupo Casos) apresentaram maior atividade eletromiográfica para os masseteres em todas as condições clínicas propostas. Na protrusão, verificou-se nos dois grupos analisados o padrão de contração eletromiográfica esperado para a manutenção desta postura que consiste em maior ativação da musculatura massetérica quando comparada com a atividade da musculatura temporal, apesar da atividade eletromiográfica ser bem maior no Grupo Casos.

Baseados nos conceitos de ativação muscular neuroanatômica para a movimentação lateral da mandíbula, sabe-se que na condição clínica de lateralidade deve ocorrer maior atividade eletromiográfica do músculo temporal do lado em que está ocorrendo à excursão da mandíbula (lado de trabalho), enquanto que para o músculo masseter, o lado mais ativado é o contra-lateral à direção desta excursão (KRAUS et al., 1994; BERSANI et al., 2011; Da SILVA et al., 2011; De ROSSI et al., 2013). Este dado foi observado nos resultados deste trabalho, em todos os grupos analisados. Mesmo com a presença de toro mandibular, houve uma tendência dos padrões de contração muscular serem semelhantes aos do Grupo Controles.

A máxima atividade eletromiográfica dos músculos masseter e temporal foi observada durante o apertamento dental em indivíduos portadores de dentição completa e sem disfunções (GIBBS et al., 1984; BLANKSMA; VAN EIJDEN, 1995), resultados concordes aos desta pesquisa para os dois grupos analisados, com uma hiperativação dos músculos masseteres para o Grupo Casos. 
O aumento da atividade eletromiográfica sugere estímulo da musculatura massetérica dos indivíduos portadores de toro mandibular para conseguirem manter as posições posturais.

A mastigação é uma das funções mais importantes desenvolvidas pelo sistema estomatognático, considerada um complexo e sofisticado mecanismo motor neurofisiológico, o primeiro passo dentro do processo digestório (ORCHARDSON; CADDEN, 1998), com atividade neuromuscular integrada com a contração sincrônica dos músculos responsáveis pela elevação, abaixamento, lateralidade e protrusão da mandíbula (MOYERS, 1991). A manutenção dos arcos dentais, como a oclusão e sua estabilidade e principalmente com o estímulo funcional sobre o periodonto, músculos e articulações são fundamentais para a mastigação (MOLINA, 1989; VAN DER BILT, 2002).

Os dentes são importantes para o sistema mastigatório (POLZER et al., 2010) e, fatores oclusais tais como mudança no padrão de contato oclusal pode gerar assimetria na função dos músculos da mastigação (FERRARIO et al., 1999, ADHIKARI et al., 2011). Nosso trabalho evidenciou por meio dos resultados obtidos que o grupo formado por indivíduos com toro mandibular apresentaram padrão de ativação muscular massetérica alterado, sugerindo que a presença do toro promove alterações na função muscular.

A mastigação tem a finalidade de fragmentar o alimento em partículas menores, preparando-o para a deglutição e digestão, sendo uma função fisiológica cujo processo de aprendizagem tem início com a erupção dos primeiros dentes decíduos (NASCIMENTO et al., 2011) e é dependente de um complexo sistema integrado por músculos, ligamentos, estruturas ósseas e dentes, controlado pelo sistema nervoso central (COELHO-FERRAZ, 2009). O ato de mastigar envolve atividades de vários músculos da face, dentre os quais, destacam-se os músculos masseteres e os temporais, que tem participação ativa durante o processo de trituração do alimento (RAHAL; GOFFI-GOMEZ, 2009). 
Existem vários fatores que influenciam a função mastigatória de forma a alterá-la. Esses fatores podem envolver alterações estruturais, como por exemplo a presença de toro mandibular ou funcionais do sistema estomatognático, que desencadeiam um desequilíbrio, do qual um dos sinais pode ser a disfunção mastigatória (WHITAKER, 2009).

A análise da mastigação por meio da eletromiografia têm sido utilizada por vários autores como Hermens et al., 1999; Bazzotti, 1999; Buzinelli e Berzin, 2001; Gonzalez et al., 2002; Tomiyama et al., 2004, Siéssere et al., 2009, e os dados obtidos com estes trabalhos são considerados importantes fontes de informações clínicas e análises cinesiológica funcional do sistema estomatognático (GLAROS et al., 1997; SGOBBI; BERZIN, 1998; RILO et al., 1997; MICHELOTTI et al., 1997).

Em 1997, De Luca, verificou a necessidade de diminuir as influências das características não-estacionárias do sinal eletromiográfico nos resultados obtidos durante condições clínicas dinâmicas, limitando a análise de sinais em contrações não-isométricas repetitivas, como a mastigação, a períodos de sinal semelhantes aos observados em contrações isométricas. Em função disto é que a análise da eficiência do ciclo mastigatório por meio da integral da envoltória do sinal eletromiográfico vem ao encontro, sendo esta uma medida matemática usada para avaliar desempenho e eficiência, e estabelecer comparações entre indivíduos em atividades dinâmicas, não estacionárias, como a mastigação (SIÉSSERE et al., 2009; BERSANI et al., 2011; Da SILVA et al., 2011; De ROSSI et al., 2013). Os dados obtidos analisam a área sob a curva do sinal eletromiográfico dos ciclos mastigatórios e os valores são em microvolts/segundo. Segundo o SENIAM (European Recommendations for Surface Electromyography) e o guia para reportar dados eletromiográficos (HERMENS et al., 1999), esta é a melhor maneira de analisar eficiência de um processo fisiológico dinâmico como a mastigação. Esta forma de análise da função mastigatória é considerada inovadora, com vantagens em relação ao método de peneiragem fragmentada, como a simplicidade, 
velocidade, precisão, reprodutibilidade, higiene, além de propiciar medir um grande número de amostras rapidamente, por isso foi objeto da escolha para analisar o desempenho dos ciclos mastigatórios entre os grupos dessa pesquisa.

O desempenho mastigatório apresenta grandes alterações com a perda dos dentes, dependendo das condições da dentição, que inclui o número de dentes presentes em oclusão funcional, o número de dentes posteriores em contato, a extensão das áreas funcionais de contato, o grau de maloclusão, as parafunções e alterações anatômicas intra-orais (HELKIMO et al., 1978), dessa forma, um dos critérios de inclusão dessa amostra era a presença de todos os dentes, com exceção dos terceiros molares.

A avaliação da atividade mioelétrica tem se constituído em uma importante ferramenta para a investigação das bases fisiopatológicas das alterações que acometem o desempenho da musculatura facial durante a mastigação (REGALO et al. 2006a). A função mastigatória, por meio da dinâmica mandibular fisiológica, propicia estímulos mecânicos adequados à cabeça da mandíbula e ligamento periodontal, principais meios de adaptação para o crescimento e desenvolvimento facial, assim como para a manutenção dos processos adaptativos, presentes por toda a vida (PLANAS, 1987; MCNEILL, 2000). Enlow, em 1993 verificou que os indivíduos adquirem um comportamento mastigatório e que o mesmo promove alterações estruturais e funcionais subsequentes no esqueleto facial. Dessa forma, este trabalho teve o objetivo de avaliar se a presença do toro poderia influenciar a função mastigatória e observouse que o desempenho mastigatório apresentou uma diferença drástica tanto na mastigação de alimento macios, como uvas passas, alimentos duros, como o amendoim e a mastigação nãohabitual, controlada, de parafilme em ambas as arcadas. Foi possível verificar uma hiperativação muscular dos masseteres e temporais para os indivíduos com toro executarem a mesma função mastigatória que o grupo controle. 
A atuação da musculatura da mastigação é influenciada por inúmeros fatores, sendo que muitos deles não estão totalmente estabelecidos, como por exemplo, a presença do toro mandibular e palatino. Assim, salienta-se a necessidade da realização de estudos futuros que enfoquem padrões morfológicos faciais diferentes, comparação entre idade, gênero, estudos longitudinais para o controle da função mastigatória e muscular. 
CONCLUSÕES 



\section{CONCLUSÕES}

Diante dos resultados obtidos no presente trabalho foi possível concluir que:

$\checkmark$ Ocorreu um aumento da atividade EMG nos músculos masseter e temporal dos Grupos Casos em relação aos Controles.

$\checkmark$ A presença de toro mandibular está associada a alterações no padrão de ativação da musculatura mastigatória nas condições posturais da mandíbula e na mastigação. 


\section{REFERÊNCIAS}





\section{REFERÊNCIAS}

1. Adhikari H, Kapoor A, Prakash U. Electromyographic pattern of masticatory muscles in altered dentition. Part II. J Conserv Dent 2011; 14:120-127.Al-Bayaty HF, Murti PR, Matthews R, Gupta PC. An epidemiological study of tori among 667 dental outpatients in Trinidad \& Tobago, West Indies. Int Dent J 2001; 51(4):300-304.

2. Antoniades DZ, Belazi M, Panayiotis P. Concurrence of torus palatinus with palatal and buccal exostoses-Case report and review of the literature. Oral Surg Oral Med Oral Pathol Oral Radiol Endod 1998; 85:552-557.

3. Apinhasmit W, Jainkittivong A, Swasdison S. Torus palatinos and torus mandibularis in Thai population. Science Asia, 2002, 28:105-111.

4. Arita CA. Análise eletromiográfica e eletrognatográfica da Estimulação Elétrica Transcutânea (T.E.N.S.) em pacientes com dor-disfunção miofascial - efeitos sobre os valores da distância interoclusal e posição de repouso mandibular. 2000. Dissertação (Mestrado) - Faculdade de Odontologia de Ribeirão Preto da Universidade de São Paulo; Ribeirão Preto.

5. Basmajian JV, de Luca CJ. Muscles alive: their function revealed by electromyography. 5ed. Baltimore: Williams and Wilkins, 1985:276-282.

6. Bazzotti L. Electromyography tension and frequency spectrum analysis of some masticatory muscles at rest, isotonic and isometric contractions. Electromyogr Clin Neurophysiol., v.39, n.7, pp.421-34, 1999.

7. Bernaba JM. Morphology and incidence of torus palatinus and mandibularis in Brasilian Indians. J Dent Res. 1977 May;56(5):499-501.

8. Blankasma NG, Van Eijden TM. Electromyographic heterogeneity in the human temporalis and masseter muscles during static biting, open/close excursions, and chewing. J. Dent. Res., v.74, n.6, p.1318-1327, jun. 1995.

9. Bukhari S, Qasi SS, Awan TM, Azad AA, Zeeshan M. Prevalence of torus palatinus among 300 indonesian patients. Pakistan Oral \& Dent J 2007; 27:89-92.

10. Buzinelli RV, Berzin F. Electromyographic analysis of fatigue in temporalis and masseter muscles during continuous chewing. J. Oral Rehabil., v.28, n.12, p.11651167, dec. 2001. 
11. Bersani E, Regalo SC, Siéssere S, Santos CM, Chimello DT, De Oliveira RH, Semprini M. Implant-supported prosthesis following Brånemark protocol on electromyography of masticatory muscles. J Oral Rehabil. 2011 Sep;38(9):668-73.

12. Çagirankaya LB, Hatipoglu MG, Kansu O. Is there in association between torus mandibularis and bite force? Hace Dishekimligi Fakultesi Dergisi Cilt 2005, 29:1517.

13. Canto, G. D. L. Prevalência de tórus mandibular em diferentes faixas etárias. 2009. Monografia (Pós-Graduação em Odontologia) - Universidade Federal de Santa Catarina, Florianópolis.

14. Carr AB. Postural contractile activities of human jaw muscles following use of an occlusal splint. J. Oral Rehabil., v. 18, p. 185-191, 1991.

15. Cecílio, F.A.; Regalo, S.C.; Palinkas, M.; Issa, J.P.; Siéssere, S.; Hallak, J.E.; Machado-De-Sousa, J.P.; Semprini, M. Ageing and surface EMG activity patterns of masticatory muscles. J Oral Rehabil, v. 37, n. 4, p. 248-255, 2010.

16. Chong SS, Ouyang G, Tian WG. Power spectral analysis of electromyografic signal of mastigatory muscles at rest position and habitual clench. J. P. D., v.65, n.4, p.553556, 1991.

17. Clifford T, Fartash L. Mandibular tori, migrane and temporomandibular disorders. British Dent J, 1996, 180: 382-384.

18. Coelho-Ferraz, MJP, Bérzin, Fausto ; Amorim, Cesar ; Queluz, Dagmar de Paula . Electromyographic Evaluation of Mandibular Biomechanic. International Journal of Morphology (Online), v. 27, p. 485-490, 2009.

19. Cooper BC. The role of bioelectronic instrumentation in the documentation and management of temporomandibular disorders. Oral Surg. Oral Med. Oral Pathol. Oral Radiol. Endod., v.83, n.1, p.91-100, 1997.

20. Da Silva RJ, Issa JP, Semprini $M$, da Silva $\mathrm{CH}$, de Vasconcelos PB, Celino CA, Siéssere S, RegaloSC. Clinical feasibility of mandibular implant overdenture retainers submitted to immediate load. Gerodontology. 2011 Sep;28(3):227-32.

21. De Rossi M, Santos CM, Migliorança R, Regalo SC. All on Four ${ }^{\circledR}$ Fixed Implant Support Rehabilitation: A Masticatory Function Study. Clin Implant Dent Relat Res. 2013 Jan 10. 
22. De Luca C. J. The use of surface electromyography in biomechanics. J Appl. Biomech. 1997; 13: 135-163.

23. Dosumu O, Arotiba JT, Ogunyinka AO. The prevalence of palatine and mandibular tori in a nigerian population. Odont Stomatol Tropic 1998, 21:6-8.

24. Eggen S, Natvig B. Relationship between torus mandibularis and number of present teeth. Scan J Dent Res 1986; 94(3): 233-240.

25. Eggen S. Torus mandibularis: an estimation of the degree of genetic determination. Acta Odontol Stan.1989; 47(6):409-415.

26. Enlow DH. Crescimento facial. 3.ed. São Paulo: Artes Médicas, 1993.

27. Ferrario VF, Sforza C, D’Aaddona A, Barbini E. Electromyographic activity of muscles in normal young people: Statistical evaluation of reference values for clinical applications. J. Oral Rehabi., v.20, p.271-280, 1993.

28. Ferrario VF, Sforza C, Serrão G. The effects of a single intercuspal interference on electromyographic characteristics of human masticatory muscles during maximal voluntary teeth clenching. Cranio 1999; 17:184-188.

29. Ferrario VF, Serrao G, Dellavia C, Caruso E, Sforza C. Relationship between the number of occlusal contacts and masticatory muscle activity in healthy young adults. J. Craniomand. Pract., v.20, n.2, p.91-98, 2002.

30. Furtado ACN, Leite AKM, Albuquerque RA, Sobral APU. Correlação entre a presença de exostoses e disfunção temporomandibular. RBPS 2008, 21: 174-179.

31. García-García AS, Martínez-González JM, Gómez-Font R, Soto-Rivadeneira A, Oviedo-Roldán L. Current status of the torus palatinus and torus mandibularis. Med Oral Patol Oral Cir Bucal. 2010;15(2):e353-360.

32. Gibbs CH, Mahan PE, Wilkinson TM, Mauderli A. EMG activity of the superior belly of the lateral pterygoid muscle in relation to other jaw muscles. J. Prosthet. Dent., v.51, n.5, p.691-702, may. 1984.

33. Glaros AG, Glass EG, Brokman D. Electromyographic data from TMD patients with miofascial pain and from matched control subjects: evidence for statistical, not clinica significance. J Orofac Pain, v.11, n.2, p.125-129, 1997. 
34. González R, Sifre S, Benedito J, Nogués V. Comparison of electromyographic pattern of sensory experts and untrained subjects during chewing of Mahon cheese. $\mathrm{J}$ Dairy Res., v.69, n.1, p.151-61, 2002.

35. Grandi G, Maito FDM, Rados PU, Santana Filho M. Estudo epidemiológico das lesões ósseas diagnosticadas no serviço de patologia bucal da PUCRS. Rev Cir Traumatol Buco-maxilo Fac 2005; 5: 67-74.

36. Harper RP, Bruin H, Burcea I. Muscle activity during mandibular movements in normal and mandibular retrognathic subjects. J. Oral Maxillofac. Surg., v. 55, n. 3, p. 225-233, 1997.

37. Hassan KS, Alagl AS, Abdel-Hady A. Torus mandibularis bone chips combined with platelet rich plasma gel for treatment of intrabony osseous defects: clinical and radiographic evaluation. Int J Oral Maxillofac Surg 2012. doi.org/10.1016/ j.ijom.2012.02.009.

38. Helkimo E, Carlsson GE, Helkimo M. Chewing efficiency and state of dentition. A methodologic study. Acta Odontol Scand 1978; 36(1):33-41.

39. Hermens HJ, Freriks B, Merletti R. European recommendations for surface electromyography - results of the SENIAM project. v. 8, 1999. p. 13-58.

40. Houssay AB. Nervous and hormonal control of the structure and I-131 uptake by the submaxillaries. Ala J Med Sci; 3(3): 312-24, 1966 Jul.

41. Igarashi $\mathrm{Y}$, Ohzeki S, Uesu K, Nakabayashi T, Kanazawa E. Frequency of mandibular tori in the present-day Japanese. Anthropologic Science 2008, 116:17-32.

42. Jankelson RR. Scientific rationale for surface electromyography to measure postural tonicity in dental patients. Cranio., v.8, n.3, p.207-209, jul. 1990.

43. Kerdpon D, Sirirungrojying S. A clinical study of oral tori in southern Thailand: prevalence and relation parafunctional activity. Eur J Oral Sci. 1999;107(1): 9-13.

44. Kolas S, Halperin V, Jefferis K, Huddleston S, Robinson HB. The occurrence of torus palatines an torus mandibularis in 2.478 dental patients. Oral Surg Oral Med Oral Pathol. 1953;6(9):1134-41. 
45. König JR., B, Vitti M. A eletromiografia aplicada ao campo odontológico. ARS CVRANDI Odontol., v.1, n.4, p.16-19, 1974.

46. Kraus WE, Torgan CE.; Taylor, D. A. Skeletalmuscles adaptation to chronic low frequency motor nerve stimulation. Exerc. Sport Sci. Rev., v. 22. p. 313-360, 1994.

47. Landulpho AB, Silva WA, Vitti M. Electromyographic evaluation of masseter and anterior temporalis muscles in patients with temporomandibular disorders following interocclusal appliance treatment. J. Oral Rehabil., v. 31, n. 2, p. 95-98, 2004.

48. Liu ZJ, Yamagata K, Kasahara, Y, Ito G. Electromyographic examination of jaw muscles in relation to symptoms and occlusion of patients with temporomandibular joint disorders. J. Oral Rehab., v.26, n.1, p.33-47, 1999.

49. Marchiori SC, Vitti M. Estudo eletromiográfico do músculo orbicular da boca. R. G. O., Porto Alegre, v.44, n.6, p.331-334, 1996.

50. Martins MD, Lata SP, Martins MAT, Bussadori SK, Fernandes K. Toro palatino e mandibular: revisão de literatura. Cons Scientiae Saúde 2007; 6:57-62.

51. McNeill C. Ciência e Prática da Oclusão. Trad. AJG Perrotti. São Paulo: Quintessence; 2000.

52. Michelotti A, Farella M, Vollaro S, Martina R. Mandibular rest position and electrical activity of the masticatory muscles. J Prosthet Dent; 78(1): 48-53, 1997 Jul.

53. Molina OF. Fisiopatologia craniomandibular. São Paulo: Pancast, 1989.

54. Morrison MD, Tamimi F. Oral Tori Are Associated With Local Mechanical and Systemic Factors: A Case-Control Study. J Oral Maxillofac Surg. 2012 Sep 22. pii: S0278-2391(12)01148-2. doi: 10.1016/j.joms.2012.08.005.

55. Moyers RE. Temporomandibular muscle contraction patterns in angle class II, division I malocclusions: an electromyographic analysis. Am. J. Orthodont., v. 35, p. 837-857, 1949.

56. Moyers RE. Maturação da musculatura orofacial. In: Ortodontia. Rio de Janeiro: Guanabara Koogan, 1991. p. 64-85. 
57. Nakamura K, Masumi S, Andoh S, Takeuchi T, Kubo M, Yasomoto K. Realtionships between mandibular torus and occlusal force, occlusal contact area and parafuncion. The J of Kyushu Dental Society 2007.

58. Nascimento GKBO, Cunha DA, Lima LM, Moraes KJR, Pernambuco LA, Régis RMFL, Silva HJ. Eletromiografia de superfície do músculo masseter durante a mastigação: uma revisão sistemática. Rev. CEFAC, São Paulo. 2011.

59. Nascimento Filho E, Seixas MT, Mazzoni A, Weckx LLM. Osteomas exofíticos múltiplos de ossos craniofaciais não associados à Síndrome de Gardner: relato de caso. Rev Bras de Otorrino 2004, 70:836-839.

60. Neville BW, Damm DD, Allen CM, Bouquot JE. Patologia oral e maxilofacial. $2^{\mathrm{a}}$ ed. Rio de Janeiro: Guanabara Koogan; 2004:798.

61. Okeson JP. Tratamento das desordens temporomandibulares e oclusão. São Paulo: Artes Médicas; 2000.

62. Orchardson R, Cadden SW. The scientific basis of eating. In: Mastication. London: Linden R.W.A., 1998. p.76-121.

63. Pancherz H. Temporal and masseter muscle activity in children and adults with normal occlusion. Acta Odontol. Scand., v.38, n.6, p.343-348, 1980.

64. Pechenkina EA, Benfer Jr. RA. The role of occlusal stress and gengival infection in the formation of exostoses on mandible and maxilla from Neolithic China. Homo. 2002;53 (2):112-130.

65. Pinho JC, Caldas FM, Mora MJ, Santana-Penin U. Electromyographic activity in patients with temporomandibular disorders. J. Oral Rehabil., v.27, n.11, p.985-990, 2000.

66. Pinzón RT. Prevalencia de torus palatino y mandibular em los pacientes que assistieron a las clínicas de la facultad de odontología de la universidad de cartagena durante el periodo 1995-2004. 2007

67. Ponzoni, D. et al. Remoção cirúrgica de toro palatino para confecção de prótese total convencional - indicações de diferentes incisões. Rev. F. Odont., v.13, p.66-70, 2008. 
68. Planas P. La thérapeutique orthodontique la plus precoce. Avec les composites polymérisables avec l’ultra-violet. L’Orthodontie Française, v.48, p.117-185, 1977.

69. Polzer I, Schimmel M, Müller F et al. Edentulism as part of the general health problems of elderly adults. Int Dent J 2010; 60:143-155.

70. Rahal A, Goffi-Gomez MVS: Electromyographic study of the masseter muscle during maximal voluntary clenching and habitual chewing in adults with normal occlusion. Rev soc bras fonoaudiol 2009;14:160-164.

71. Rancan, S.V.; Bataglion, C.; Bataglion, S.A.; Bechara, O.M.; SepriniI, M.; Siéssere, S.; Sousa, J.P.; Crippa, J.A.; Hallak, J.E.; Regalo, S.C. Acupuncture and temporomandibular disorders: a 3-month follow-up EMG study. J Altern Complement Med, v. 15, n. 12, p. 1307-1310, 2009.

72. Regalo SCH, Vitti M, Hallak JEC, Semprini M, Mattos MG, Tosello DO, Constancio RF, Pegoraro ME, Lopes RA. EMG analysis of the upper and lower fascicles of the orbicularis oris muscle in deaf individuals. Electromyog. Clin. Neurophysiol., v.43, n.6, p.367-372, 2003.

73. Regalo SC, Vitti M, Hallak JE, Siessere S, Pagnano VO, Semprini M. Electromyographic analysis of upper and lower fascicles of the orbicularis oris muscle in deaf individuals, in mandibular rest position, compared to hearers. Electromyog. Clin. Neurophysiol., v.46, n.4, p.211-215, jul.-aug. 2006a.

74. Regalo SCH, Vitti M, Semprini M, Rosa LB, Martinez FH, Santos CM, Hallak JEC. Electromyographic analysis of the masseter and temporal muscles in oralized deaf individuals. Electromyog. Clin. Neurophysiol., v. 46, n.4, p.217-222,jul.-aug. 2006b.

75. Regalo SC, Santos CM, Vitti M, Regalo CA, Vasconcelos PB, Mestriner JRW, Semprini, M, Dias FJ, Hallak JE, Siéssere S. Evaluation of molar and incisor bite force in indigenous compared with white population in Brazil.Arch. Oral Biol.,v.53, n.3, p.282-286, mar. 2008.

76. Regezi JA, Sciubba JJ. Patologia bucal - correlações clinicopatológicas. $3^{\mathrm{a}}$ ed. Rio de Janeiro, Guanabara Koogan, 2000:309.

77. Renon M, Isolan TMP, Zambrano CMP, Campos C, Dalmagro Filho L. Toro palatino e mandibular: um estudo morfológico em pacientes e cabeças ósseas. RGO. 1994;42(3):176-178. 
78. Rilo B, Santana U, Mora MJ, Cadarso CM. Myoelectrical activity of clinical rest position and jaw muscle activity in Young adults. J Oral Rehabil 1997 Out; 24(10): 735-40.

79. Santos CM, Vitti M, Matsumoto W, Berro RJ, Semprini M, Hallak JEC, et al. Using overdenture on implants and complete denture: effects on postural maintenance of masticatory musculature. Braz J Oral Sci 2008 Abr-Jun; 7(25): 1550-1554.

80. Serra VBR, Campos MIC, Guimarães JP. Prevalência de toro mandibular e palatino em pacientes bruxômanos. Rev Serv ATM 2005, 5:20-25.

81. Serra VBR, Campos MIC. Correlação entre bruxismo e toro mandibular e palatino: caso clínico. Rev Serv ATM 2005, 5, 60-63.

82. Sgobbi de Faria CRS, Bérzin F. Electromyographic study of the temporal, masseter and suprahyoid muscle in mandibular rest position. J. Oral Rehabil., v. 25, n. 10, p. 776-780, 1998.

83. Shankland WE 2nd. Anterior throat pain syndromes: causes for undiagnosed craniofacial pain. Cranio 2010; 28:50-59.

84. Shimahara T, Ariyoshi Y, Nakajima Y, Shimara M, Kurisu Y, Tsuji M. Mandibular torus with tongue movement disorder: a case report. Bullet of the Osaka Medic Coll 2007; 53:143-46.

85. Siéssere S, Lima Nde S, Semprini M, Sousa LG, Issa JPM, Monteiro SAC, et al. Masticatory processe in individuals with maxillary and mandibular osteoporosis: electromyographic analysis. Osteoporos Int. 2009 Nov; 20(11): 1847-51.

86. Simões WA. Ortopedia funcional dos maxilares. 3 ed., São Paulo: Artes Médicas, 2003. 1024p.

87. Sirirungrojying S, Kerdpon D. Relationship between oral tori and temporomandibular disorders. Int Dent J. 1999;49 (2):101-104.

88. Springer J. Tori mandibular with speech impediment. Oral Surg Oral Med Oral Pathol 1954; 7:1270-2.

89. Takasugi Y, Shiba M, OkamotoS, Hatta K, Koga Y. Difficult laryngoscopy caused by massive mandibular tori. J of Anesthesia 2009; 23:278-80. 
90. Thompson H. Occlusion in clinical practice: dental practitioner handbook. Wright P. S. G., v. 30, p. 4-5, 1981.

91. Tomiyama N, Ichida T, Yamaguchi K. Electromyographic activity of lower lip muscles when chewing with the lips in contact and apart. Angle Orthod 2004

92. Trenouth MJ. Muscle response to Twin-block appliance. Am. J. Orthod. Dentofac. Orthop., v. 117, n. 4, p. 25, 2000.

93. Ueda MH, Ishizuka Y, Miyamoto K, Morimoto N, Tanne K. Relationship between masticatory muscle activity and vertical craniofacial morphology. Angle Orthod., v.68, n.3, p.233-238, 1998.

94. Van Der Bilt, A. Human oral function: a review. Braz. J. Oral Sci. , v. 1, n. 1, p. 7-18, 2002.

95. Vitti M, Basmajian JV. Muscles of mastication in small children: an electromyographic analysis. Am. J. Orthod., v.68, n.4, p.412-419, 1975.

96. Vitti M, Basmajian JV. Integrated actions of masticatory muscles: simultaneous EMG from eight intramuscular electrodes. Anat. Rec., v.187, n.2, p.173-190, feb. 1977.

97. Voudouris JC, Kuftinic MM. Improved clinical use of twin-block and herbst as a result of radiating viscoelastic tissue forces on the condyle and fossa in treatment and long term retention: growth relativity. Am. J. Orthod. Dentofacial Orthop., v. 117. n. 3, p. 247-266, 2000.

98. Whittaker P. Laser acupuncture: past, present, and future Lasers Med Scien 2004;19: 69-80.

99. Yemm R, Nördstrom SH. Forces developed by tissue elasticity as a determinant of mandibular resting posture at the rat. Arch. Oral Biol., v.19, p.347-351, 1974. 
ANEXO 



\section{ANEXO}

ANEXO I- Parecer do Comitê de Ética

\section{UNIVERSIDADE ESTADUAL DE MONTES CLAROS - UNIMONTES}

\section{PARECER CONSUBSTANCIADO DO CEP}

\section{DADOS DO PROJETO DE PESQUISA}

Título da Pesquisa: EFEITO DO TÓRUS MANDIBULAR NA MUSCULATURA DO SISTEMA ESTOMATOGNÁTICO

Pesquisador: JOSÉ MENDES DA SILVA

Área Temática:

Versão: 1

CAAE: 13350013.6 .0000 .5146

Instituição Proponente:Universidade Estadual de Montes Claros - UNIMONTES

Patrocinador Principal: Financiamento Próprio

\section{DADOS DO PARECER}

Número do Parecer: 226.704

Data da Relatoria: 22/02/2013

\section{Apresentaçäo do Projeto:}

Considerando-se relevante a influência da musculatura na funcionalidade do sistema estomatognático, o estudo caso-controle pretende avaliar o comportamento e atuação da musculatura mastigatória frente ao tórus mandibular. $O$ termo tórus designa excrescência óssea convexa bem definida, de crescimento lento, composta por uma densa cortical, escassa em osso esponjoso, coberta por uma capa de mucosa delgada e pobremente irrigada e é considerado congênito, benigno. Denominado também de exostose. Será efetuado um exame clínico físico e bucal, coletado os dados eletromiográficos normalizados e as medidas das espessuras musculares, eficiência mastigatória, força de mordida e aplicado um questionário de qualidade de vida aos sujeitos participantes do estudo.

\section{Objetivo da Pesquisa:}

Avaliar de forma comparativa o comportamento e atuação da musculatura mastigatória frente a indivíduos portadores de tórus mandibular.

\section{Avaliaçäo dos Riscos e Benefícios:}

Será realizado um exame clínico físico e bucal, onde o indivíduo terá que ficar um tempo com a boca aberta, podendo causar certo desconforto. Destaca-se como benefícios a possibilidade de identificar se indivíduos com tórus mandibular apresentam alteraçöes na musculatura do sistema estomatognático e relação direta com a atividade eletromiográfica, eficiència mastigatória,

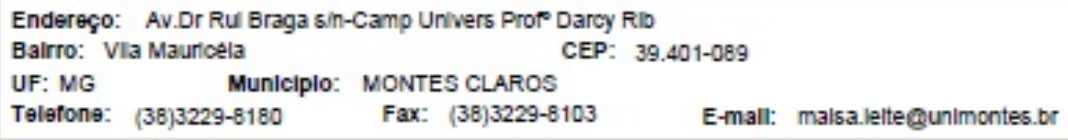




\title{
UNIVERSIDADE ESTADUAL DE MONTES CLAROS - UNIMONTES
}

espessura muscular, força de mordida,qualidade de vida e xerostomia.

Comentários e Considerações sobre a Pesquisa:

Estudo relevante na área da saúde bucal, uma vez que possa produzir resultados com influência no diagnóstico e terapêutica relativas a funcionalidade do sistema estomatognático.

Considerações sobre os Termos de apresentação obrigatória:

Termos adequados.

Recomendaçöes:

Recomenda-se enviar relatório final ao Comitê de Ética em Pesquisa.

Conclusöes ou Pendências e Lista de Inadequações:

Projeto aprovado.

Situaçäo do Parecer:

Aprovado

Necessita Apreciação da CONEP:

Não

Considerações Finais a critério do CEP:

O projeto respeita os preceitos éticos da pesquisa em seres humanos, sendo assim somos favoráveis à aprovação do mesmo.

MONTES CLAROS, 22 de Março de 2013

\author{
Assinador por: \\ Maisa Tavares de Souza Leite \\ (Coordenador)
}

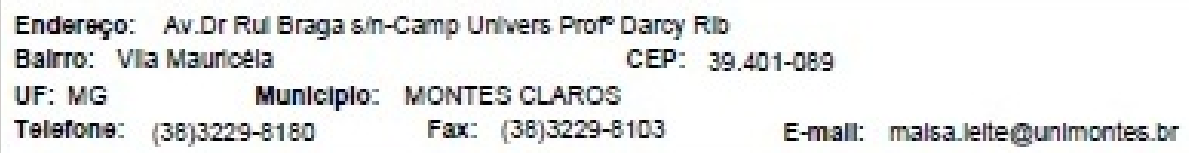

